\title{
Some remarks on the size of tubular neighborhoods in contact topology and fillability
}

\author{
KLAUS NIEDERKRÜGER \\ FRANCISCO PRESAS
}

\begin{abstract}
The well-known tubular neighborhood theorem for contact submanifolds states that a small enough neighborhood of such a submanifold $N$ is uniquely determined by the contact structure on $N$, and the conformal symplectic structure of the normal bundle. In particular, if the submanifold $N$ has trivial normal bundle then its tubular neighborhood will be contactomorphic to a neighborhood of $N \times\{\mathbf{0}\}$ in the model space $N \times \mathbb{R}^{2 k}$.

In this article we make the observation that if $\left(N, \xi_{N}\right)$ is a 3-dimensional overtwisted submanifold with trivial normal bundle in $(M, \xi)$, and if its model neighborhood is sufficiently large, then $(M, \xi)$ does not admit a symplectically aspherical filling.
\end{abstract}

57R17; 53D35

In symplectic geometry, many invariants are known that measure in some way the "size" of a symplectic manifold. The most obvious one is the total volume, but this is usually discarded, because one can change the volume (in case it is finite) by rescaling the symplectic form without changing any other fundamental property of the manifold. The first non-trivial example of an invariant based on size is the symplectic capacity (see Gromov [15]). It relies on the fact that the size of a symplectic ball that can be embedded into a symplectic manifold does not only depend on its total volume but also on the volume of its intersection with the symplectic 2-planes.

Contact geometry does not give a direct generalization of these invariants. The main difficulties stem from the fact that one is only interested in the contact structure, and not in the contact form, so that the total volume is not defined, and to make matters worse the whole Euclidean space $\mathbb{R}^{2 n+1}$ with the standard structure can be compressed by a contactomorphism into an arbitrarily small open ball in $\mathbb{R}^{2 n+1}$.

A more successful approach consists in studying the size of the neighborhood of submanifolds. This can be considered to be a generalization of the initial idea since contact balls are just neighborhoods of points. In the literature this idea has been pursued by looking at the tubular neighborhoods of transverse circles. Let $\left(N, \alpha_{N}\right)$ be a closed contact manifold. The product $N \times \mathbb{R}^{2 k}$ carries a contact structure given as 
the kernel of the form $\alpha_{N}+\sum_{j=1}^{k}\left(x_{j} d y_{j}-y_{j} d x_{j}\right)$, where $\left(x_{1}, \ldots, x_{k}, y_{1}, \ldots, y_{k}\right)$ are the coordinates of the Euclidean space. If $\left(N, \alpha_{N}\right)$ is a contact submanifold of a manifold $(M, \alpha)$ that has trivial (conformal symplectic) normal bundle, then one knows by the tubular neighborhood theorem that $N$ has a small neighborhood in $M$ that is contactomorphic to a small neighborhood of $N \times\{\mathbf{0}\}$ in the product space $N \times \mathbb{R}^{2 k}$.

The contact structure on a solid torus $V$ in $\mathbb{S}^{1} \times \mathbb{R}^{2}$ depends in an intricate way on the radius of $V$ (see Eliashberg [6]): The solid tori $\mathbb{S}^{1} \times \mathbb{D}_{R}$, and $\mathbb{S}^{1} \times \mathbb{D}_{R^{\prime}} \subset$ $\left(\mathbb{S}^{1} \times \mathbb{R}^{2}, d \theta+r^{2} d \phi\right)$ are contactomorphic if and only if $\frac{1}{R^{2}}-\frac{1}{\left(R^{\prime}\right)^{2}} \in \mathbb{Z}$. Later, examples of transverse knots in 3-manifolds were found whose maximal neighborhood is only contactomorphic to a small disk bundle in $\mathbb{S}^{1} \times \mathbb{R}^{2}$ (see Etnyre and Honda [11]). This is proved by measuring the slope of the characteristic foliation on the boundary of these neighborhoods.

A different approach has been taken by Eliashberg, Kim and Polterovich [8]. There it has been shown that a solid torus of large radius around $\mathbb{S}^{1} \times\{\mathbf{0}\}$ in $\mathbb{S}^{1} \times \mathbb{R}^{2 k}$ cannot be "squeezed" into a solid torus of small radius, if $k \geq 2$. However note that squeezing in the context of [8] is different from the naive definition, and refers to the question of whether one subset of a contact manifold can be deformed by a global contact isotopy into another one.

The present article is based on the observation that sufficiently large neighborhoods of $N \times\{\boldsymbol{0}\}$ in $N \times \mathbb{R}^{2 k}$ contain a generalized plastikstufe (for a definition of the GPS see Section 3) if $N$ is an overtwisted 3-manifold. The construction of a GPS in a tubular neighborhood is explained in Section 4. In Section 5, we show that the existence of a GPS implies nonfillability, and so we can deduce the main result of this article (Corollary 13) that an overtwisted contact manifold that is embedded into a fillable manifold cannot have a "large" neighborhood.

Unfortunately, the definition of "large" is rather subtle and does not lead to a numerical invariant, because such an invariant would depend on the contact form on the submanifold. One could simply multiply any contact form $\alpha_{N}+\sum_{j=1}^{k}\left(x_{j} d y_{j}-y_{j} d x_{j}\right)$ by a constant $\lambda>0$, and then rescale the radii in the plane by a transformation $r_{j} \mapsto r_{j} / \sqrt{\lambda}$ to change such a numerical invariant.

\section{Acknowledgments}

During the work for this article, K Niederkrüger was postdoc at the ENS de Lyon funded by the project Symplexe 06-BLAN-0030-01 of the Agence Nationale de la Recherche (ANR). 
Several people helped us writing this article: Many ideas and examples are due to Emmanuel Giroux. Example 5 was found in discussions with Hansjörg Geiges. We thank Ana Rechtman Bulajich for helping us clarifying the general ideas of the paper, and Mohammed Abouzaid and Pierre Py for extremely valuable discussions about holomorphic curves. Paolo Ghiggini and Patrick Massot pointed out to us known results about neighborhoods of transverse knots.

We would also like to thank the referee for reading carefully our article, and suggesting many improvements.

\section{Examples}

First we give an easy example that shows that embedding an overtwisted 3-manifold into a higher dimensional fillable contact manifold does not pose a fundamental problem. Embedding a contact 3-manifold into a contact manifold of dimension 7 or higher restricts by using the $h$-principle (see Gromov [16] and Eliashberg-Mishachev [9, Section 12.3]) and a general position argument to a purely topological question.

Example 1 (a) Let $M$ be an arbitrary orientable closed 3-manifold. Its unit cotangent bundle $\mathbb{S}\left(T^{*} M\right) \cong M \times \mathbb{S}^{2}$ has a contact structure defined by the canonical 1-form $\lambda_{\text {can }}$. The cotangent bundle $T^{*} M$ together with the form $d \lambda_{\text {can }}$ is an exact symplectic filling (and in fact, it can even be turned into a Stein filling).

Any non-vanishing 1 -form $\beta$ on $M$ can be normalized so that $\|\beta\|=1$ everywhere, and defines then a section $\sigma_{\beta}: M \rightarrow \mathbb{S}\left(T^{*} M\right)$ with $\sigma_{\beta}^{*} \lambda_{\text {can }}=\beta$. If $\alpha$ is a normalized contact form on $M$, then $\sigma_{\alpha}: M \rightarrow \mathbb{S}\left(T^{*} M\right)$ defines an contact embedding. This means that every (and in particular also every overtwisted) contact 3-manifold can be embedded into a Stein fillable contact 5-manifold.

(b) Now we will show that every contact 5-manifold $(M, \alpha)$ contains closed overtwisted submanifolds of dimension 3 (see also Remark 2). The proof works in two steps.

The 3-sphere $\mathbb{S}^{3} \subset \mathbb{C}^{2}$ supports an overtwisted contact structure given as the kernel of the 1 -form

$$
\alpha_{-}=i \sum_{j=1}^{2}\left(z_{j} d \bar{z}_{j}-\bar{z}_{j} d z_{j}\right)-i(f d \bar{f}-\bar{f} d f)
$$

with $f\left(z_{1}, z_{2}\right)=z_{1}^{2}+z_{2}^{2}$. In Example 5, we will describe some of the properties of $\left(\mathbb{S}^{3}, \alpha_{-}\right)$in more detail. For now consider the map

$$
\Phi: \mathbb{S}^{3} \hookrightarrow \mathbb{C}^{3},\left(z_{1}, z_{2}\right) \mapsto\left(z_{1}, z_{2}, \bar{f}\left(z_{1}, z_{2}\right)\right) .
$$


It clearly is an embedding, because the projection onto the first two coordinates in $\mathbb{C}^{3}$ already is. We will show that its image lies in a hypersurface of $\mathbb{C}^{3}$ contactomorphic to the standard contact 5-sphere. The pull-back of the standard Liouville form $\alpha_{0}=i \sum_{j=1}^{3}\left(z_{j} d \bar{z}_{j}-\bar{z}_{j} d z_{j}\right)$ of $\mathbb{C}^{3}$ gives back $\Phi^{*} \alpha_{0}=\alpha_{-}$. We have to show that $\Phi\left(\mathbb{S}^{3}\right)$ is contained in the boundary of a star-shaped domain in $\mathbb{C}^{3}$ centered at $\mathbf{0}$, because the restriction of the Liouville form to such a boundary is contactomorphic to the standard contact structure on the 5-sphere. With the help of the function $F: \mathbb{S}^{5} \rightarrow \mathbb{R},\left(z_{1}, z_{2}, z_{3}\right) \mapsto \sqrt{1+\left|z_{3}\right|^{2}}$ we can define a smooth embedding of the 5-sphere

$$
\left(z_{1}, z_{2}, z_{3}\right) \mapsto F\left(z_{1}, z_{2}, z_{3}\right) \cdot\left(z_{1}, z_{2}, z_{3}\right),
$$

that bounds a star-shaped domain. This submanifold contains the image of $\Phi$, and thus we have shown that the standard contact 5-sphere admits a contact embedding of $\left(\mathbb{S}^{3}, \alpha_{-}\right)$.

Since $\left(\mathbb{S}^{2 N-1}, \alpha_{0}\right)$ with one point removed is contactomorphic to $\mathbb{R}^{2 N-1}$ with standard contact structure (see for example Geiges [12, Proposition 2.13]) and since it is possible to embed the whole $\mathbb{R}^{2 N-1}$ into an arbitrary small Darboux chart (see for example Chekanov-van Koert-Schlenk [4, Proposition 3.1]), it follows that every 5-dimensional contact manifold $(M, \alpha)$ contains embeddings of the overtwisted 3-sphere $\left(\mathbb{S}^{3}, \alpha_{-}\right)$.

Remark 2 Atsuhide Mori proved that all contact 3-manifolds can be immersed by a contactomorphism into the standard 5-sphere [20]. He told us that if the 3-manifold in question admits a compatible planar open book decomposition, then his construction even yields an embedding. In particular, by a result of J Etnyre [10], every overtwisted manifold has a planar open book decomposition, so that every overtwisted contact manifold can be embedded into a 5-dimensional Darboux chart.

The next examples show that contact submanifolds can have infinitely large tubular neighborhoods.

Example 3 (a) Let $(M, \alpha)$ be an arbitrary contact manifold, and let $\left(\mathbb{S}^{2 n-1}, \xi_{0}\right)$ be the standard contact sphere. If $\operatorname{dim} M=2 n+2 k-1$, then it is easy to give a contact embedding

$$
\left(\mathbb{S}^{2 n-1} \times \mathbb{R}^{2 k}, \alpha_{0}+\sum_{j=1}^{k}\left(x_{j} d y_{j}-y_{j} d x_{j}\right)\right) \hookrightarrow(M, \alpha) \text {. }
$$


The proof works in two steps. For the embedding (to avoid confusion, we use below the notation $\alpha_{0}(N)$ for the standard contact form on the $(2 N-1)-$-sphere)

$\left(\mathbb{S}^{2 n-1} \times \mathbb{R}^{2 k}, \alpha_{0}(n)+\sum_{j=1}^{k}\left(x_{j} d y_{j}-y_{j} d x_{j}\right)\right) \hookrightarrow\left(\mathbb{S}^{2 n+2 k-1}, \alpha_{0}(n+k)\right)$

simply use the map

$\left(z_{1}, \ldots, z_{n} ; x_{1}, y_{1}, \ldots, x_{k}, y_{k}\right) \mapsto \frac{\left(z_{1}, \ldots, z_{n}, x_{1}+i y_{1}, \ldots, x_{k}+i y_{k}\right)}{\sqrt{1+\|\mathbf{x}\|^{2}+\|\mathbf{y}\|^{2}}}$,

where $\mathbf{x}=\left(x_{1}, \ldots, x_{k}\right)$ and $\mathbf{y}=\left(y_{1}, \ldots, y_{k}\right)$. As in Example 1(b), use the fact that $\left(\mathbb{S}^{2 N-1}, \alpha_{0}\right)$ with one point removed is contactomorphic to the standard contact Euclidean space $\mathbb{R}^{2 N-1}$, and since it is possible to embed the whole $\mathbb{R}^{2 N-1}$ into an arbitrary small Darboux chart, it follows that every $(2 n+2 k-1)$ dimensional contact manifold $(M, \alpha)$ contains embeddings of

$$
\left(\mathbb{S}^{2 n-1} \times \mathbb{R}^{2 k}, \alpha_{0}+\sum_{j=1}^{k}\left(x_{j} d y_{j}-y_{j} d x_{j}\right)\right) .
$$

(b) A generalization is obtained by choosing a contact manifold $\left(N, \alpha_{N}\right)$ that has an exact symplectic filling $(W, \omega=d \lambda)$. The Liouville field $X_{L}$ on $W$ (see Section 2.1) is globally defined, and we can use its negative flow to find an embedding of the lower half of the symplectization $(-\infty, 0] \times N$ into $W$ such that $\lambda$ pulls back to $e^{t} \alpha_{N}$. The manifold $\mathbb{S}^{1} \times W$ together with the $1-$ form $d \theta+\lambda$ is a contact manifold.

The standard model $\left(N \times \mathbb{R}^{2}, \alpha_{N}+r^{2} d \phi\right)$ can be glued outside the 0 -section onto $\mathbb{S}^{1} \times W$, and this construction yields a closed contact manifold that contains the embedding of $N \times \mathbb{R}^{2}$. This example represents an open book with binding $N$, page $W$, and trivial monodromy.

Not much is known about the different contact structures on $\mathbb{R}^{2 n+1}$ for $n \geq 2$. There exist the standard contact structure $\xi_{0}$, and many different constructions to produce structures that are not isomorphic to the standard one (for example Bates-Peschke [1], Muller [21] and Niederkrüger [22]). Unfortunately we do not have any effective techniques to decide whether these exotic contact structures are different from each other. A contact structure $\xi$ on $\mathbb{R}^{2 n+1}$ is called standard at infinity (see Eliashberg [7]), if there exists a compact subset $K$ of $\mathbb{R}^{2 n+1}$ and a number $R>0$ such that $\left(\mathbb{R}^{2 n+1}-K, \xi\right)$ is contactomorphic to $\left(\mathbb{R}^{2 n+1}-\mathbb{D}_{R}, \xi_{0}\right)$ for a closed disk of radius $R$. A contact structure $\xi$ on $\mathbb{R}^{2 n+1}$ only admits a one-point compactification to a contact structure on the sphere, if $\xi$ is standard at infinity. For most exotic contact structures it is not known 
whether they are standard at infinity or not. The only exception known so far to us was given in Niederkrüger-van Koert [23], where by removing one point from the sphere, we obtained an exotic contact structure $\xi_{P S}$ on $\mathbb{R}^{2 n+1}$ that is standard at infinity (but see also Example 5 for a further example on $\mathbb{R}^{5}$ ). A rather crude way of producing a contact structure that is not standard at infinity consists in taking the standard structure on $\mathbb{R}^{2 n+1}$, and do the connected sum at every point $(0, \ldots, 0, k) \in \mathbb{R}^{2 n+1}$ with $k \in \mathbb{Z}$ with the sphere $\left(\mathbb{S}^{2 n+1}, \xi_{P S}\right)$. Corollary 13 yields a much nicer and very explicit way to construct an exotic contact structure that is not standard at infinity.

Example 4 The contact manifold

$$
\left(\mathbb{R}^{3} \times \mathbb{C}^{k}, \alpha_{-}+\sum_{j=1}^{k} r_{j}^{2} d \theta_{j}\right),
$$

where $\left(r_{j}, \theta_{j}\right)$ are polar coordinates on the $j$-th factor of $\mathbb{C}^{k}$, contains a GPS, and by Corollary 13 it cannot be embedded into any $(2 k+3)$-dimensional contact manifold that has an exact filling. In particular it does not embed into the standard sphere, and is hence not contactomorphic to the standard contact structure on $\mathbb{R}^{2 k+3}$. Let $K \subset \mathbb{R}^{3} \times \mathbb{C}^{k}$ be an arbitrary compact subset. Again it is easy to see that $\left(\mathbb{R}^{3} \times\right.$ $\left.\mathbb{C}^{k}-K, \alpha_{-}+\sum_{j} r_{j}^{2} d \theta_{j}\right)$ also contains a GPS, so it cannot be embedded into a "punctured" set $U-\{p\} \subset\left(\mathbb{R}^{2 k+3}, \alpha_{0}\right)$ with the standard contact structure. It follows that $\left(\mathbb{R}^{3} \times \mathbb{C}^{k}, \alpha_{-}+\sum_{j} r_{j}^{2} d \theta_{j}\right)$ is "non standard at infinity".

Let $(M, \alpha)$ be a closed contact manifold that contains a contact submanifold $N$ of codimension 2 with trivial normal bundle. A $k$-fold contact (cyclic) branched covering over $M$ consists of a closed manifold $\widetilde{M}$, and a smooth surjective map $f: \widetilde{M} \rightarrow M$ such that the map $f$ is a $k$-fold covering over $M-N$, and there is an open neighborhood $\widetilde{U} \subset \widetilde{M}$ of $f^{-1}(N)$ diffeomorphic to $N \times \mathbb{D}_{\epsilon}$, and a neighborhood $U \subset M$ of $N$ diffeomorphic to $N \times \mathbb{D}_{\epsilon^{k}}$ such that the map $f$ can be written as

$$
f: N \times \mathbb{D}_{\epsilon} \rightarrow N \times \mathbb{D}_{\epsilon^{k}},(p, z) \mapsto\left(p, z^{k}\right),
$$

when restricted to $\tilde{U}$ (see Gromov [16] or Geiges [13, Section 7.5]).

Using the branched covering, it is easy to define a contact structure on $\widetilde{M}$. First isotope $\alpha$ in such a way that it takes the form $\left.\alpha\right|_{T N}+r^{2} d \phi$ on a subset $N \times \mathbb{D}_{\delta^{k}} \subset N \times \mathbb{D}_{\epsilon^{k}}$ for some $\delta$ with $0<\delta<\epsilon$. The pull-back $\widetilde{\alpha}:=f^{*} \alpha$ defines on $\widetilde{M}$ a $1-$ form that satisfies everywhere away from $f^{-1}(N)$ the contact property. In a neighborhood of $f^{-1}(N)$ we find a subset contained in $\widetilde{U}$ that is diffeomorphic to $N \times \mathbb{D}_{\delta}$ on which $\tilde{\alpha}$ evaluates to $\left.\alpha\right|_{T N}+k r^{2 k} d \phi$. 
Remove the fiber $N \times\{0\}$ from $\tilde{U}$ and glue in $N \times \mathbb{D}_{\delta^{k}}$ via the map $F:\left(p, r e^{i \phi}\right) \mapsto$ $\left(p, \sqrt[k]{r} e^{i \phi}\right)$ along $N \times\left(\mathbb{D}_{\delta}-\{0\}\right)$. The pull-back $F^{*} \widetilde{\alpha}$ yields $\left.\alpha\right|_{T N}+k r^{2} d \phi$ on the punctured disk bundle, which we can easily extend to the whole patch we are gluing in. We denote this slightly modified contact form again by $\widetilde{\alpha}$. By using a linear stretch map on the disk, we finally see that the submanifold $f^{-1}(N) \cong N$ has a neighborhood in $(\widetilde{M}, \widetilde{\alpha})$ that is of size $\sqrt{k} \delta^{k}$ with respect to the model form

$$
\left(N \times \mathbb{R}^{2},\left.\alpha\right|_{T N}+r^{2} d \phi\right) .
$$

Example 5 (a) There is an interesting contact structure on the odd dimensional spheres $\mathbb{S}^{2 n-1} \subset \mathbb{C}^{n}$ given as the kernel of the 1 -form

$$
\alpha_{-}=i \sum_{j=1}^{n}\left(z_{j} d \bar{z}_{j}-\bar{z}_{j} d z_{j}\right)-i(f d \bar{f}-\bar{f} d f)
$$

with $f\left(z_{1}, \ldots, z_{n}\right)=z_{1}^{2}+\cdots+z_{n}^{2}$. This form is compatible with the open book with binding $B=f^{-1}(0)$, and fibration map $\theta=\bar{f} /|f|$. In abstract terms, this contact structure is given by the open book with page $P \cong T^{*} \mathbb{S}^{n-1}$ and monodromy map corresponding to the negative Dehn-Seidel twist.

An interesting feature of these spheres is that they can be stacked into each other via the natural inclusions $\mathbb{S}^{3} \hookrightarrow \mathbb{S}^{5} \hookrightarrow \mathbb{S}^{7} \hookrightarrow \cdots$ respecting the contact form, and that $\left(\mathbb{S}^{3}, \alpha_{-}\right)$is overtwisted.

We find a contact branched cover $f: \mathbb{S}^{5} \rightarrow\left(\mathbb{S}^{5}, \alpha_{-}\right)$given by

$$
f\left(z_{1}, z_{2}, z_{3}\right)=\frac{\left(z_{1}, z_{2}, z_{3}^{k}\right)}{\left\|\left(z_{1}, z_{2}, z_{3}^{k}\right)\right\|}
$$

that is branched along $\mathbb{S}^{3}$. By choosing $k$ large enough, we will obtain with the construction described above a contact structure on $\mathbb{S}^{5}$ that contains an embedding of $\left(\mathbb{S}^{3}, \alpha_{-}\right)$with large neighborhood. According to Corollary 13 and Theorem 11, this contact structure will not admit a symplectically aspherical filling.

This result is not completely satisfactory, since we do not get an explicit value for $k$. In fact, we expect that $\left(\mathbb{S}^{3}, \alpha_{-}\right)$already has a large neighborhood in all of the spheres $\left(\mathbb{S}^{2 n-1}, \alpha_{-}\right)$so that taking a branched covering should not really be necessary (the non-fillability has been proved by Bourgeois and van Koert [3] using contact homology).

(b) A second class of examples is obtained by embedding an overtwisted contact 3-manifold $(M, \alpha)$ into its unit cotangent bundle $\mathbb{S}\left(T^{*} M\right)$ as explained in Example 1(a). If the embedding $\sigma_{\alpha}$ is homotopic to a constant section $M \times\left\{p_{0}\right\}$ in the trivialization $\mathbb{S}\left(T^{*} M\right) \cong M \times \mathbb{S}^{2}$, then we can construct a $k$-fold covering 
of the unit cotangent bundle branched over $\pm \sigma_{\alpha}$ by simply covering the $2-$ sphere $k$-times. Note that the diffeomorphism type of the manifold does not change, but for sufficiently large $k$, the covering will not be symplectically aspherically fillable, in contrast to the covered manifold.

\section{Preliminaries}

\subsection{Fillability}

In this section, we will briefly present some standard definitions and properties regarding fillability and $J$-holomorphic curves.

Definition A Liouville field $X_{L}$ is a vector field on a symplectic manifold $(W, \omega)$ for which $\mathcal{L}_{X_{L}} \omega=\omega$.

If $(W, \omega)$ is a symplectic manifold with boundary $M:=\partial W$, and if $X_{L}$ is a Liouville field defined in a neighborhood of $\partial W$ that is transverse to $M$, then the kernel of the $1-$ form

$$
\alpha:=\left.\left(\iota_{X_{L}} \omega\right)\right|_{T M}
$$

defines a contact structure on $M$.

Definition A symplectic manifold $(W, \omega)$ is called symplectically aspherical if

$$
\int_{\mathbb{S}^{2}} u^{*} \omega=0
$$

for any smooth map $u: \mathbb{S}^{2} \rightarrow W$.

Definition Let $(M, \xi)$ be a closed contact manifold. A compact symplectic manifold $(W, \omega)$ with boundary $\partial W=M$ is called a strong (symplectic) filling of $(M, \xi)$, if there exists a Liouville field $X_{L}$ in a neighborhood of the boundary $M$ pointing outwards through $M$ such that $\left.\left({ }_{{ } X_{L}} \omega\right)\right|_{T M}$ defines a contact form for $\xi$. If $(W, \omega)$ is symplectically aspherical, we call it a symplectically aspherical filling, and if the vector field $X_{L}$ is defined globally on $W$, we speak of an exact symplectic filling.

In particular it follows that an exact symplectic filling is also symplectically aspherical.

Remark 6 In a symplectic filling, we can always find a neighborhood of $M$ that is of the form $(-\epsilon, 0] \times M$ by using the flow $\Phi_{t}$ of $X_{L}$ to define

$$
(-\epsilon, 0] \times M \rightarrow W,(t, p) \mapsto \Phi_{t}(p) .
$$


Denote the hypersurfaces $\{t\} \times M$ by $M_{t}$, and the 1 -form $\iota_{X_{L}} \omega$ by $\hat{\alpha}$. It is clear that $\hat{\alpha}$ defines on every hypersurface $M_{t}$ a contact structure. The Reeb field $X_{\text {Reeb }}$ is the unique vector field on $(-\epsilon, 0] \times M$ that is tangent to the hypersurfaces $M_{t}$, and satisfies both $\omega\left(X_{\text {Reeb }}, Y\right)=0$ for every $Y \in T M_{t}$, and $\omega\left(X_{L}, X_{\text {Reeb }}\right)=1$. This field restricts on any hypersurface $M_{t}$ to the usual Reeb field for the contact form $\left.\hat{\alpha}\right|_{T M_{t}}$. Below we will show that the "height" function $h:(-\epsilon, 0] \times M \rightarrow \mathbb{R},(t, p) \mapsto t$ is plurisubharmonic with respect to certain almost complex structures.

In the context of this article we will use the term "adapted almost complex structure" in the following sense.

Definition Let $(W, \omega)$ be a symplectic filling of a contact manifold $(M, \alpha)$. An almost complex structure $J$ is a smooth section of the endomorphism bundle $\operatorname{End}(T W)$ such that $J^{2}=-\mathbf{1}$. We say that $J$ is adapted to the filling, if it is compatible with $\omega$ in the usual sense, which means that for all $X, Y \in T_{p} W$

$$
\omega(J X, J Y)=\omega(X, Y)
$$

holds, and

$$
g(X, Y):=\omega(X, J Y)
$$

defines a Riemannian metric. Additionally, we require $J$ to satisfy close to the boundary $M=\partial W$ the following properties: For the two vector fields $X_{L}$ and $X_{\text {Reeb }}$ introduced above, $J$ is defined as

$$
J X_{L}=X_{\text {Reeb }} \quad \text { and } \quad J X_{\text {Reeb }}=-X_{L},
$$

and $J$ leaves the subbundle $\xi_{t}=T M_{t} \cap \operatorname{ker} \hat{\alpha}$ invariant.

Proposition 7 Let $U$ be an open subset of $\mathbb{C}$, and let $u: U \rightarrow(-\epsilon, 0] \times M$ be a $J$-holomorphic map. The function $h \circ u: U \rightarrow \mathbb{R}$ is subharmonic.

Proof A short computation shows that $\hat{\alpha}=-d h \circ J$, and then we get

$$
\begin{aligned}
0 & \leq u^{*} \omega=u^{*} d \iota_{X_{L}} \omega=u^{*} d \widehat{\alpha}=u^{*} d(-d h \circ J)=-u^{*} d d^{c} h \\
& =-d d^{c}(h \circ u)=\left(\frac{\partial^{2} h \circ u}{\partial x^{2}}+\frac{\partial^{2} h \circ u}{\partial y^{2}}\right) d x \wedge d y
\end{aligned}
$$

which completes the proof.

If $u: \Sigma \rightarrow(-\epsilon, 0] \times M$ is a $J$-holomorphic curve that lies in a level set $h^{-1}(t)$ of the height function, then the image of $u$ is everywhere tangent to $\xi_{t}$, and so its energy $E(u)=\int_{\Sigma} u^{*} \omega=\int_{\partial \Sigma} u^{*} \hat{\alpha}$ vanishes, which implies that $u$ has to be constant. 
Corollary 8 By the strong maximum principle and the boundary point lemma (for example Gilbarg and Trudinger [14]), any connected $J$-holomorphic curve $u:(\Sigma, \partial \Sigma) \rightarrow$ ( $W, \partial W$ ) is either constant or it touches $M=\partial W$ only at its boundary $\partial \Sigma$, and this intersection is transverse. Furthermore, if $u$ is non constant, then the boundary curve $\left.u\right|_{\partial \Sigma}$ has to intersect the contact structure $\xi$ on $\partial W$ in positive Reeb direction.

Let $\phi: N \hookrightarrow \rightarrow M$ be an immersion of a manifold $N$ in $M$. We define the self-intersection set of $N$ as

$$
N_{\varnothing}:=\left\{p \in N \mid \exists p^{\prime} \neq p \text { with } \phi(p)=\phi\left(p^{\prime}\right)\right\} .
$$

\subsection{The tubular neighborhood theorem for contact submanifolds}

Let $N$ be a contact submanifold of $(M, \alpha)$. The contact structure $\xi=\operatorname{ker} \alpha$ can be split along $N$ into the two subbundles

$$
\left.\xi\right|_{N}=\xi_{N} \oplus \xi_{N}^{\perp}
$$

where $\xi_{N}$ is the contact structure on $N$ given by $\xi_{N}=\left.T N \cap \xi\right|_{N}=\left.\operatorname{ker} \alpha\right|_{T N}$, and $\xi_{N}^{\perp}$ is the symplectic orthogonal of $\xi_{N}$ inside $\left.\xi\right|_{N}$ with respect to the form $d \alpha$. Note that $\xi_{N}^{\perp}$ carries a symplectic structure given by $d \alpha$, but neither $\xi_{N}^{\perp}$ nor the associated conformal symplectic structure depend on the contact form $\alpha$ chosen on $M$. The bundle $\xi_{N}^{\perp}$ can be identified with the normal bundle of $N$.

The standard neighborhood theorem for contact submanifolds states that $\xi_{N}^{\perp}$ determines a small neighborhood of $N$ completely.

Theorem 9 Let $\left(N, \xi_{N}\right)$ be a contact submanifold of both $\left(M_{1}, \xi_{1}\right)$ and $\left(M_{2}, \xi_{2}\right)$. Assume that the two normal bundles $\left(\xi_{1}\right)_{N}^{\perp}$ and $\left(\xi_{2}\right)_{N}^{\perp}$ are isomorphic as conformal symplectic vector bundles. Then there exists a small neighborhood of $N$ in $M_{1}$ that is contactomorphic to a small neighborhood of $N$ in $M_{2}$.

If $N$ has trivial conformal symplectic normal bundle $\xi_{N}^{\perp}$, then we call the product space $N \times \mathbb{R}^{2 k}$ with contact structure $\alpha_{N}+\sum_{j=1}^{k}\left(x_{j} d y_{j}-y_{j} d x_{j}\right)$ the standard model for neighborhoods of $N$.

\section{The generalized plastikstufe (GPS)}

Definition Let $(M, \alpha)$ be a $(2 n+1)$-dimensional contact manifold, and let $S$ be a closed (n-1)-dimensional manifold. A generalized plastikstufe (GPS) is an immersion

$$
\Phi: S \times \mathbb{D} \rightarrow M,\left(s, r e^{i \phi}\right) \rightarrow \Phi\left(s, r e^{i \phi}\right),
$$


such that the pull-back $\Phi^{*} \alpha$ reduces to the form $f(r) d \phi$ with $f \geq 0$ that only vanishes for $r=0$, and $r=1$. Furthermore there is an $\epsilon>0$ such that self-intersections may only happen between points of the form $\left(s, r e^{i \phi}\right)$, and $\left(s^{\prime}, r^{\prime} e^{i \phi}\right)$ with $r, r^{\prime} \in(\epsilon, 1-\epsilon)$ that have equal $\phi$-coordinate. Finally there must be an open set joining $S \times\{0\}$ with $S \times \partial \mathbb{D}$ that does not contain any self-intersection points.

We call $S \times\{0\}$ (or also its image) the core of the GPS, and $S \times \partial \mathbb{D}$ (or again the image) its boundary. We denote $S \times(\mathbb{D}-\{0\}-\partial \mathbb{D})$ by GPS* and call it the interior of the GPS.

Remark 10 The contact structure induces a foliation on GPS*, and the leaves are given by the sets $\{\phi=$ const $\}$. We are hence requiring that self-intersections only happen between points lying on the same leaf. A different way to state this requirement consists in saying that there is a continuous map

$$
\theta: \Phi\left(\mathrm{GPS}^{*}\right) \rightarrow \mathbb{S}^{1}
$$

such that $\theta\left(\Phi\left(s, r e^{i \phi}\right)\right)=\phi$.

The main implication of the existence of a GPS is given by the following theorem which we will prove in Section 5.

Theorem 11 A closed contact manifold $(M, \alpha)$ that contains a GPS does not admit a symplectically aspherical filling.

Remark 12 A more precise analysis of bubbling (as in Ivashkovich-Shevchishin [17]) should make it possible to prove that a GPS is an obstruction to finding even a (semipositive) strong symplectic filling. In Remark 15, we sketch how the proof would have to be modified. Note though that to apply [17], we would also have to require that the self-intersections of the GPS are clean.

\section{Constructing immersed plastikstufes in neighborhoods of contact submanifolds}

\subsection{Local construction in codimension two}

The overtwisted contact structure given as the standard example in the literature is $\mathbb{R}^{3}$ with the structure induced by the contact form

$$
\alpha_{-}=\cos r d z+r \sin r d \phi,
$$


written in cylindrical coordinates $(r, \phi, z)$ such that $x=r \cos \phi, y=r \sin \phi$, and $z=z$. Any plane $\{z=$ const $\}$ contains an overtwisted disk of radius $r=\pi$ centered at the origin. From the classification in Eliashberg [7], it follows that $\left(\mathbb{R}^{3}, \alpha_{-}\right)$is up to contactomorphism the unique contact structure on $\mathbb{R}^{3}$ that is overtwisted at infinity, and hence any sufficiently small contractible neighborhood of an overtwisted disk in a contact 3-manifold is contactomorphic to $\left(\mathbb{R}^{3}, \alpha_{-}\right)$.

The Reeb field $X_{\text {Reeb }}$ associated to $\alpha_{-}$is given by

$$
X_{\text {Reeb }}=\frac{1}{r+\sin r \cos r}\left(\sin r \partial_{\phi}+(\sin r+r \cos r) \partial_{z}\right) .
$$

Its flow $\Phi_{t}$ is linear, because it does not change $r$, and the coefficients of the $z$ - and the $\phi$-directions only depend on the $r$-coordinate. The Reeb field is only tangent to the overtwisted disk along the circle of radius $r_{0}$ with $r_{0}=-\tan r_{0}\left(r_{0} \approx 2.029\right)$. Inside this circle, $X_{\text {Reeb }}$ has a positive $z$-component, outside it has a negative one. This means the overtwisted disk $\mathbb{D}_{\mathrm{OT}}$ and its translation by the Reeb flow $\Phi_{t}\left(\mathbb{D}_{\mathrm{OT}}\right)$ for a time $t \neq 0$ only intersect along the circle of radius $r_{0}$ (see Figure 1). More precisely, the Reeb field reduces on the circle of radius $r_{0}$ to $X_{\text {Reeb }}=1 /\left(r_{0} \sin r_{0}\right) \partial_{\phi}$, so that it defines a rotation with period $T=2 \pi r_{0} \sin r_{0} \approx 11.4$.

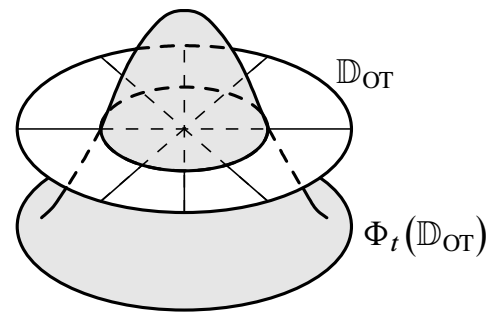

Figure 1: The overtwisted disk and its image under the Reeb flow only intersect along a circle of radius $r_{0}$.

Define now on $\mathbb{R}^{3} \times \mathbb{R}^{2}$ the contact form $\alpha_{-}+R^{2} d \theta$, where $(R, \theta)$ are polar coordinates of $\mathbb{R}^{2}$. To construct a GPS in $\mathbb{R}^{3} \times \mathbb{R}^{2}$, we will use the first step of the construction in Presas [24]. For this we interpret $\mathbb{R}^{3} \times \mathbb{R}^{2}$ as a contact fibration over the plane.

Definition Let $\pi: P \rightarrow B$ be a fiber bundle that is endowed with a hyperplane distribution $\eta \leq T P$. We call $(P, B, \pi, \eta)$ a contact fibration, if $\eta$ restricts on every fiber $F_{p}:=\pi^{-1}(p), p \in B$ to a contact structure $\xi_{p}:=T F_{p} \cap \eta$.

For more details see Lerman [18]. A contact fibration carries a natural parallel transport given as follows: Choose a $1-$ form $\beta$ on $P$ such that $\eta=\operatorname{ker} \beta$. Let $p$ be a point 
in the base manifold, and $\tilde{p} \in F_{p}$ an arbitrary point in the fiber over $p$. Then we define for a vector $v \in T_{p} B$ a unique lift $\tilde{v} \in T_{\widetilde{p}} P$ by the conditions that $D \pi(\widetilde{v})=v$, that $\tilde{v} \in \eta$, and that $d \beta(\widetilde{v}, \cdot)$ vanishes on $\xi_{p}$. One can easily check that the lift $\tilde{v}$ is independent of the choice of the 1 -form $\beta$.

Choose a path $\gamma:[0,1] \rightarrow B$ in the base manifold. The parallel transport of the fiber $F_{\gamma(0)}$ consists in lifting the vectors $\dot{\gamma}(t)$ to all the points in the corresponding fibers, and integrating the flow to obtain a 1-parameter family of maps $\Gamma_{t}: F_{\gamma(0)} \rightarrow F_{\gamma(t)}$. The main property of this construction is that $\Gamma_{t}$ induces a contactomorphism between the fibers $\left(F_{\gamma(0)}, \xi_{\gamma(0)}\right)$ and $\left(F_{\gamma(t)}, \xi_{\gamma(t)}\right)$.

It is obvious that the projection of $\left(\mathbb{R}^{3} \times \mathbb{R}^{2}, \alpha_{-}+R^{2} d \theta\right)$ onto the last two coordinates is a contact fibration. We will trace a closed path $\gamma: \mathbb{S}^{1} \subset \mathbb{C} \rightarrow \mathbb{R}^{2}$ that has the shape of a figure-eight, with the double point at the origin, and such that both loops of the eight enclose equal area with respect to the area form $2 R d R \wedge d \theta$ (twice the standard area form). Start at the origin of the disk, at $\gamma(1)=0$ on this closed loop, and consider the overtwisted disk $\mathbb{D}_{\mathrm{OT}}$ described above in the fiber $\mathbb{R}^{3} \times\{0\}$. By using the parallel transport of $\mathbb{D}_{\text {OT }}$ along the path $\gamma$, we are able to construct an immersed plastikstufe. The parallel transport reduces in the fibers to the flow of the vector field $-c X_{\text {Reeb }}$ with $c=|\gamma|^{2} d \theta(\dot{\gamma})$, so that the monodromy of a closed loop is just given by the Reeb flow $\Phi_{-T}$ for a time $T$ that is equal to the algebraic area enclosed by the loop. The total area of the figure-eight $\gamma$ vanishes, because on one part of the eight, we are turning in positive direction, on the other in the opposite one, and the area of both parts was chosen to be equal.

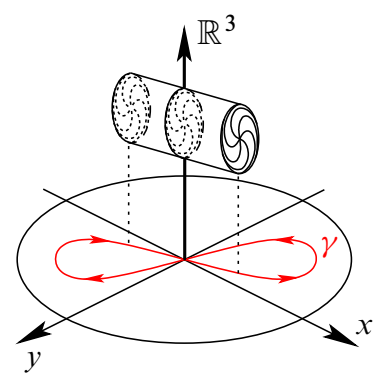

Figure 2: Parallel transport of the overtwisted disk along a figure-eight path yields an immersed plastikstufe.

We will describe the construction more explicitly to better understand the self-intersection set. The parallel transport of the overtwisted disk defines an immersion

$$
\mathbb{D}_{\mathrm{OT}} \times \mathbb{S}^{1} \hookrightarrow \mathbb{R}^{3} \times \mathbb{R}^{2},\left((x, y, 0), e^{i \theta}\right) \mapsto\left(\Phi_{-T(\theta)}(x, y, 0), \gamma\left(e^{i \theta}\right)\right),
$$


where $T\left(\theta_{0}\right)=\int_{0}^{\theta_{0}}|\gamma(s)|^{2} d \theta(\dot{\gamma}(s)) d s$. The map is well defined, because $T(\theta+$ $2 \pi)=T(\theta)$. It is also easy to see that this map is an immersion.

The only self-intersection points may lie over the crossing $\gamma(1)=\gamma(-1)$ in the figureeight, and in fact, since the Reeb flow moves the interior of the overtwisted disk up, and the outer part down, self-intersections only happen between the two circles

$$
\left\{\left(r_{0} \cos \phi, r_{0} \sin \phi, 0\right)\right\} \times\{-1,1\} \subset \mathbb{D}_{\mathrm{OT}} \times \mathbb{S}^{1} .
$$

Denote the area enclosed by one of the petals of the figure-eight path by $A$. The images of two points $\left(\left(r_{0} \cos \phi, r_{0} \sin \phi, 0\right), 1\right)$ and $\left(\left(r_{0} \cos \left(\phi-t_{0}\right), r_{0} \sin \left(\phi-t_{0}\right), 0\right),-1\right)$ are only identical if $t_{0}=A /\left(r_{0} \sin r_{0}\right)$.

If the size of $\gamma$ is chosen in such a way that $A=2 \pi r_{0} \sin r_{0}$, then pair of points for which the plastikstufe intersects itself lie on equal rays of the overtwisted disk, and we have constructed a GPS. The figure-eight path has to enclose a sufficiently large area, but such a path $\gamma$ cannot be realized in a disk $\mathbb{D}_{R}$ of radius $R<\sqrt{2 r_{0} \sin r_{0}} \approx 1.91$.

\subsection{Higher codimension}

Use the same contact structure on $\left(\mathbb{R}^{3}, \alpha_{-}\right)$as above, and extend it to a contact structure on $\mathbb{R}^{3} \times \mathbb{C}^{k}$ with contact form

$$
\alpha_{-}+\sum_{j=1}^{k} r_{j}^{2} d \phi_{j},
$$

where $\left(r_{j}, \phi_{j}\right)$ are polar coordinates for the $j$-th $\mathbb{C}$-factor in $\mathbb{C}^{k}$. Take the following $k$-fold product of figure-eight loops of different sizes, and group them into a map

$$
\Gamma: \mathbb{T}^{k} \leftrightarrow \mathbb{C}^{k},\left(e^{i \theta_{1}}, \ldots, e^{i \theta_{k}}\right) \mapsto\left(\gamma\left(e^{i \theta_{1}}\right), 2^{1 / 2} \gamma\left(e^{i \theta_{2}}\right), \ldots, 2^{(k-1) / 2} \gamma\left(e^{i \theta_{k}}\right)\right) .
$$

This map is an immersion with self-intersection set

$$
\mathbb{T}_{\check{\chi}}^{k}=\left\{\left(e^{i \theta_{1}}, \ldots, e^{i \theta_{k}}\right) \in \mathbb{T}^{k} \mid \text { at least one of the } \theta_{j} \text { lies in } \pi \mathbb{Z}\right\} .
$$

Define functions $T_{j}(\theta):=2^{j-1} \int_{0}^{\theta} \gamma^{*}\left(r^{2} d \phi\right)$, and $T\left(e^{i \theta_{1}}, \ldots, e^{i \theta_{k}}\right)=\sum_{j=1}^{k} T_{j}\left(\theta_{j}\right)$. Then the immersion

$$
\begin{aligned}
\mathbb{D}_{\mathrm{OT}} \times \mathbb{T}^{k} & \rightarrow \mathbb{R}^{3} \times \mathbb{C}^{k}, \\
\left((x, y, 0) ;\left(e^{i \theta_{1}}, \ldots, e^{i \theta_{k}}\right)\right) & \mapsto\left(\Phi_{-T\left(\theta_{1}, \ldots, \theta_{k}\right)}(x, y, 0) ; \Gamma\left(e^{i \theta_{1}}, \ldots, e^{i \theta_{k}}\right)\right),
\end{aligned}
$$

where $\Phi_{t}$ denotes the Reeb flow in $\mathbb{R}^{3}$, is a GPS. Obviously the self-intersection points of this map are contained in $\mathbb{D}_{\mathrm{OT}} \times \mathbb{T}_{\chi}^{k}$. Consider two points $\left(e^{i \theta_{1}}, \ldots, e^{i \theta_{k}}\right)$ 
and $\left(e^{i \psi_{1}}, \ldots, e^{i \psi_{k}}\right)$ that have the same image under $\Gamma$. It follows for each pair $\left(\theta_{j}, \psi_{j}\right)$ that either $\theta_{j}=\psi_{j}$ or that both $\theta_{j}, \psi_{j} \in \pi \mathbb{Z}$. The disks lying over such points are given by $\Phi_{-T(\theta)}\left(\mathbb{D}_{\mathrm{OT}}\right)$ and $\Phi_{-T(\boldsymbol{\psi})}\left(\mathbb{D}_{\mathrm{OT}}\right)$ respectively, where $\mathbb{D}_{\mathrm{OT}}=$ $\{(x, y, 0) \mid\|(x, y, 0)\| \leq \pi\}$. The Reeb flow is $\phi$-invariant and preserves the distance of the points $(x, y, 0)$ from the $z$-axis. Hence by comparing the $z$-coordinates, the equation $\Phi_{-T(\theta)}(x, y, 0)=\Phi_{-T(\boldsymbol{\psi})}\left(x^{\prime}, y^{\prime}, 0\right)$ implies for two points $(x, y, 0)$, and $\left(x^{\prime}, y^{\prime}, 0\right) \in \mathbb{D}_{\mathrm{OT}}$ with $\|(x, y, 0)\|=\left\|\left(x^{\prime}, y^{\prime}, 0\right)\right\| \neq r_{0}$, that $T(\boldsymbol{\theta})=T(\boldsymbol{\psi})$ has to hold. The coefficients chosen for the paths in $\Gamma$ are such that $T$ is injective on the subset $\left\{\left(\pi a_{1}, \ldots, \pi a_{k}\right)\right\}$ with all $a_{j} \in\{0,1\}$. This implies that $\theta=\psi$, and also $(x, y, 0)=\left(x^{\prime}, y^{\prime}, 0\right)$, so that no self-intersections can happen for points with $\|(x, y, 0)\| \neq r_{0}$.

Self-intersections of $\mathbb{D}_{\mathrm{OT}} \times \mathbb{T}^{k}$ can hence only exist for points where the distance of $(x, y)$ from the origin is equal to $r_{0}$, and because both of the disks enclosed by the figure-eight loop $\gamma$ have area $A=2 \pi r_{0} \sin r_{0}$, the holonomy will always correspond to a rotation by a multiple of $2 \pi$ so that intersecting points always lie on the same leaf of the foliation, and all conditions of a GPS are met by this immersion.

\subsection{Application to contact submanifolds}

Let $\left(N, \alpha_{N}\right)$ be an overtwisted contact 3-manifold. We will show that the product manifold $\left(N \times \mathbb{C}^{k}, \alpha_{N}+\sum_{j=1}^{k} r_{j}^{2} d \theta_{j}\right)$, where $\left(r_{j}, \theta_{j}\right)$ are polar coordinates on the $j$-th factor of $\mathbb{C}^{k}$, contains a GPS.

Consider a small contractible neighborhood of an overtwisted disk $\mathbb{D}_{\text {OT }}$ in $N$. This neighborhood is contactomorphic to $\left(\mathbb{R}^{3}, \alpha_{-}\right)$, because it is overtwisted at infinity. Choose a large ball $B$ in $\mathbb{R}^{3}$ (so large that the Reeb flow for $\alpha_{-}$restricted to the overtwisted disk exists for long enough times), then there is a function $f: N \rightarrow \mathbb{R}_{>0}$ such that the chosen ball $\left(B, \alpha_{-}\right)$can be embedded by a strict contactomorphism (that means preserving the contact form) into $\left(N, f \alpha_{N}\right)$. The contact form $f \alpha_{N}+$ $\sum_{j=1}^{k} f r_{j}^{2} d \theta_{j}$ on the product manifold $N \times \mathbb{C}^{k}$ can be transformed by the map $\left(p ; z_{1}, \ldots, z_{k}\right) \mapsto\left(p ; z_{1} / \sqrt{f}, \ldots, z_{k} / \sqrt{f}\right)$ into

$$
\left(N \times \mathbb{C}^{k}, f \alpha_{N}+\sum_{j=1}^{k} r_{j}^{2} d \theta_{j}\right) .
$$

This contains a subset of the form $\left(B \times \mathbb{C}^{k}, \alpha_{-}+\sum_{j=1}^{k} r_{j}^{2} d \theta_{j}\right)$ in which we can perform the construction explained above. 
Corollary 13 Let $(M, \alpha)$ be a closed $(2 n+1)$-dimensional contact manifold that contains an overtwisted contact 3-dimensional submanifold $N$ with trivial contact normal bundle. There is a neighborhood of $N$ that is contactomorphic to a neighborhood $U$ of $N \times\{\mathbf{0}\}$ in the product space $\left(N \times \mathbb{C}^{k}, \alpha_{N}+\sum_{j=1}^{k} r_{j}^{2} d \theta_{j}\right)$.

If the neighborhood $U$ contains a sufficiently large disk bundle of $N \times\{\mathbf{0}\}$, then it follows that $M$ does not admit a symplectically aspherical filling.

Proof By the construction just described $\left(N \times \mathbb{C}^{k}, \alpha_{N}+\sum_{j=1}^{k} r_{j}^{2} d \theta_{j}\right)$ contains a GPS. Since the GPS is compact, it is contained in some disk bundle around $N \times\{\mathbf{0}\}$. If the neighborhood of $N$ is contactomorphic to this disk bundle, then $(M, \alpha)$ contains a GPS, and hence by Theorem 11 it cannot have a symplectically aspherical filling. $\square$

Remark 14 A natural question to ask is if Corollary 13 can be modified for contact manifolds that admit the embedding of large tubular neighborhoods of $P S$-overtwisted manifolds (that means manifolds that contain a plastikstufe or even a GPS). The proof for submanifolds containing a proper plastikstufe should reduce to finding a standard neighborhood theorem for the plastikstufe, and then trying to rescale the contact form in such a way that the Reeb flow will exist for sufficiently large times. Afterward it should be straightforward to apply the same reasoning as in Section 4.

\section{Proof of Theorem 11}

\subsection{Sketch of the proof}

The proof is based on Niederkrüger [22] (which in turn is ultimately based on Eliashberg [5] and Gromov [15]), and it is very helpful to have a good understanding of this first article. Assume that $(M, \alpha)$ admits a symplectically aspherical filling $(W, \omega)$. We choose an adapted almost complex structure $J$ on $W$ that has in a neighborhood of the core $S \times\{0\}$ the special form described in [22, Section 3], and in a neighborhood of the boundary $S \times \partial \mathbb{D}$ the particular form described in Section 5.3 below.

The chosen almost complex structure allows us to explicitly write down the members of a Bishop family around the core of the GPS, so that we find a non-empty moduli space $\mathcal{M}$ of holomorphic disks $u:(\mathbb{D}, \partial \mathbb{D}) \rightarrow\left(W\right.$, GPS $\left.{ }^{*}\right)$ with a marked point $z_{0} \in \partial \mathbb{D}$. The boundary of each holomorphic disk $u$ intersects every regular leaf of the GPS exactly once, in other words the composition $\left.\theta \circ u\right|_{\partial \mathbb{D}}: \mathbb{S}^{1} \rightarrow \mathbb{S}^{1}$ defines a diffeomorphism on the circle. The image of the Bishop family in the moduli space is canonically diffeomorphic to a neighborhood of the core $S \times\{0\}$ via the evaluation map

$$
\mathrm{ev}_{z_{0}}: \mathcal{M} \rightarrow \mathrm{GPS}^{*}, u \mapsto u\left(z_{0}\right) .
$$


We can apply similar intersection arguments for the boundary $S \times \partial \mathbb{D}$ of the GPS (Section 5.3), and for the core ([22, Section 3]), showing that there exists a neighborhood of $\partial$ GPS that has empty intersection with any holomorphic disk, and that the only disks that come close to the core are the ones lying in the Bishop family.

Choose now a smooth generic path $\gamma \subset S \times \mathbb{D}$ that avoids the self-intersection points of the GPS, and that connects the core $S \times\{0\}$ with the boundary of the GPS. In Section 5.2, we prove that the moduli space $\mathcal{M}_{\gamma}:=\mathrm{ev}_{z_{0}}^{-1}(\gamma)$ is a smooth 1-dimensional manifold. From now on, we will further restrict $\mathcal{M}_{\gamma}$ to the connected component of the moduli space that contains the Bishop family. Then in fact $\mathcal{M}_{\gamma}$ has to be diffeomorphic to an open interval. The compactification at one of the ends of the interval simply consists in decreasing the size of the disks in the Bishop family until they collapse to the point $\gamma(0)$ on the core of the GPS.

Our aim will be to understand the possible limits at the other end of the interval $\mathcal{M}_{\gamma}$, and to deduce a contradiction to the fillability of $M$. The energy of all disks $u \in \mathcal{M}_{\gamma}$ is bounded by $2 \pi \max f$, where $\alpha=f(r) d \phi$ on the GPS. By requiring that the GPS has only clean intersections, we could apply the compactness theorem in IvashkovichShevchishin [17] to deduce even a contradiction for the existence of a semipositive filling (see Remark 15). Instead of merely referring to that result, we have decided to give a full proof of compactness in our situation that shows that no type of bubbling is possible (see Section 5.4). This way we can drop the stringent conditions on the self-intersections of the GPS, and the required proof is in fact significantly shorter than the full proof of the compactness theorem.

It then follows that for any sequence of disks $u_{k} \in \mathcal{M}_{\gamma}$, we find a family of reparametrizations $\phi_{k}: \mathbb{D} \rightarrow \mathbb{D}$ such that $u_{k} \circ \phi_{k}$ contains a subsequence converging uniformly with all derivatives to a disk $u_{\infty} \in \mathcal{M}_{\gamma}$. This means that the closure of $\mathcal{M}_{\gamma}$ is compact, and in fact homeomorphic to a closed interval, but since we know at the same time that the limit element $u_{\infty}$ lies in $\mathcal{M}_{\gamma}, u_{\infty}$ has a small neighborhood in $\mathcal{M}_{\gamma}$ homeomorphic to an open interval, it follows that $u_{\infty}$ is not a boundary point of $\mathcal{M}_{\gamma}$. Compactness contradicts thus the existence of the filling.

Remark 15 We will briefly sketch how [17] could be used to prove even the nonexistence of a semipositive filling, if the GPS is cleanly immersed.

The limit of a sequence of holomorphic disks can be described as the union of finitely many holomorphic spheres $u_{S}^{1}, \ldots, u_{S}^{K}$ and finitely many holomorphic disks $v_{1}, \ldots, v_{N}$. The holomorphic disks $v_{j}:(\mathbb{D}, \partial \mathbb{D}) \rightarrow(W$, GPS $)$ are everywhere smooth with the possible exception of boundary points that lie on self-intersections of the GPS. Here $v_{j}$ will still be continuous though (this behavior can be visualized by taking the 
baby-example of a figure-eight path in the complex plane $\mathbb{C}$. By the Riemann mapping theorem, there is a holomorphic disk enclosed into each of the loops, but these disks cannot be smooth on their boundary at the self-intersection point of the eight).

We will now first prove that the limit curve of a sequence in $\mathcal{M}_{\gamma}$ is only composed of a single disk, which then necessarily has to be smooth. Assume we would have a decomposition into several disks $v_{1}, \ldots, v_{N}$. The boundary of each of these disks $v_{j}$ is a continuous path in GPS*, we can hence combine the disks with the projection $\theta$ defined in Remark 10 to obtain a continuous map $\left.\theta \circ v_{j}\right|_{\partial \mathbb{D}}: \mathbb{S}^{1} \rightarrow \mathbb{S}^{1}$. Thus we can associate to each of the disks $v_{j}$ a degree. In fact it follows that $\left.\operatorname{deg} \theta \circ v_{j}\right|_{\partial \mathbb{D}}>$ 0 , because almost all points on the boundary of $v_{j}$ are smooth, and for them $v_{j}$ has to intersect, by Corollary 8, all leaves of the foliation of the GPS in positive direction. Finally assume that there are still several disks, each one necessarily with $\left.\operatorname{deg} \theta \circ v_{j}\right|_{\partial \mathbb{D}} \geq 1$. This means that the composition of the maps $\left.\theta \circ v_{j}\right|_{\partial \mathbb{D}}$ will cover the circle several times, but this is not possible for the limit of injective maps $\left.\theta \circ u_{k}\right|_{\partial \mathbb{D}}$. There is hence only a single disk in the limit. Using Theorem 19 below it finally also follows that this disk is smooth, and has a boundary that lifts to a smooth loop in $S \times \mathbb{D}$.

The reason why there are no holomorphic spheres as bubbles is a genericity argument. Since the disk and all spheres are regular smooth holomorphic objects, we can compute the dimension of the moduli space in which the bubble tree would lie. By the assumption of semi-positivity, it follows that the dimension would be negative.

\subsection{The moduli space}

The aim of this section is to define the moduli space of holomorphic disks and to prove that it is a smooth manifold. Care has to be taken, because the boundary condition considered in this article is not a properly embedded, but only an immersed submanifold. The main idea is to restrict to those holomorphic curves whose boundary lies locally on embedded subsets of the immersed submanifold. We can then easily adapt standard results.

Let $(W, J)$ be an almost complex manifold, and let $L$ be a compact manifold with $\operatorname{dim} L=1 / 2 \operatorname{dim} W$.

Definition An immersed totally real submanifold is an immersion $\phi: L \uparrow W$ such that

$$
\left(D \phi \cdot T_{x} L\right) \oplus\left(J \cdot D \phi \cdot T_{x} L\right)=T_{\phi(x)} W
$$

at every $x \in L$. 
Let $\phi: L \uparrow \rightarrow W$ be a totally real immersed submanifold with self-intersection set $L_{\varnothing}$, and let $\Sigma$ be a Riemann surface with $N$ boundary components $\partial \Sigma_{1}, \ldots, \partial \Sigma_{N}$. Then define $\mathcal{B}(\Sigma ; L)$ to be the set of Sobolev $W^{k, p}$-maps with $k \geq 1$ and $p>2$ (by the Sobolev embedding theorem these maps are continuous)

$$
u:(\Sigma, \partial \Sigma) \rightarrow(W, \phi(L))
$$

for which the boundary circles $\left.u\right|_{\partial \Sigma}$ can be lifted to continuous loops $u^{L}: \partial \Sigma \rightarrow L$ such that $\phi \circ u^{L}=\left.u\right|_{\partial \Sigma}$, and such that each of the $N$ components of $u^{L}$ intersects the complement of $L_{\varnothing}$.

Note that with our conditions the lift of the boundary circles $\left.u\right|_{\partial \Sigma}$ is unique, because if there were two different loops $u^{L}, \widehat{u}^{L}: \partial \Sigma_{j} \rightarrow L$ with $\phi \circ u^{L}=\phi \circ \widehat{u}^{L}$, both $u^{L}\left(\partial \Sigma_{j}\right)$ and $\hat{u}^{L}\left(\partial \Sigma_{j}\right)$ would intersect the complement of $L_{\varnothing}$, and then it follows that the set $\left\{z \in \partial \Sigma_{j} \mid u^{L}(z)=\widehat{u}^{L}(z)\right\}$ is non-empty. Furthermore this set is closed, because it is the preimage of the diagonal $\triangle_{W}:=\{(p, p) \mid p \in W\}$ under the map $u^{L} \times \widehat{u}^{L}: \partial \Sigma_{j} \times \partial \Sigma_{j} \rightarrow W \times W$ intersected with the diagonal $\triangle_{\partial \Sigma_{j}}$. Finally, $L$ can be covered with open sets on each of which the immersion $\phi$ is injective, and hence if $u^{L}(z)=\widehat{u}^{L}(z)$ there is also an open neighborhood of $z$ on which both paths coincide. It follows that $u^{L}$ and $\widehat{u}^{L}$ are equal.

We have to prove that $\mathcal{B}(\Sigma ; L)$ is a Banach manifold by finding a suitable atlas. To define a chart around a map $u_{0} \in \mathcal{B}(\Sigma ; L)$, construct first a Banach space $B_{u_{0}}$ by considering the space of $W^{k, p}$-sections $\sigma$ in $E:=u_{0}^{*} T W$ satisfying the following boundary condition: Take the unique collection of loops $u_{0}^{L}$ such that $\phi \circ u_{0}^{L}=\left.u_{0}\right|_{\partial \Sigma}$. We can define a subbundle $F \leq\left. E\right|_{\partial \Sigma}$ over the boundary of the surface by pushing $T_{u_{0}^{L}(z)} L$ with $D \phi$ into $E$. We require the sections $\sigma: \Sigma \rightarrow E$ to lie along the boundary $\partial \Sigma$ in the subbundle $F$.

Our aim will be to map these sections in a suitable way into $\mathcal{B}(\Sigma ; L)$. For this, choose first an embedding $\widetilde{\phi}$ of $L$ into some Euclidean space $\mathbb{R}^{N}$, and lift then the immersion $\phi: L \rightarrow W$ to a map $\phi \times \tilde{\phi}: L \hookrightarrow W \times \mathbb{R}^{N}$ whose image we will denote by $\tilde{L}$ (see Figure 3).

Introduce on a small collar neighborhood of the boundary of the domain $\Sigma$ the coordinates $(t, p) \in(-\epsilon, 0] \times \partial \Sigma$, and choose a smooth function $F: \Sigma \rightarrow[0,1]$ whose support is contained in this collar $(-\epsilon, 0] \times \partial \Sigma$, and that is equal to 1 in a smaller neighborhood of $\partial \Sigma$. We can easily lift maps $u:(\Sigma, \partial \Sigma) \rightarrow(W, \phi(L))$ in $\mathcal{B}(\Sigma ; L)$ to maps

$$
\tilde{u}:(\Sigma, \partial \Sigma) \rightarrow\left(W \times \mathbb{R}^{N}, \tilde{L}\right)
$$




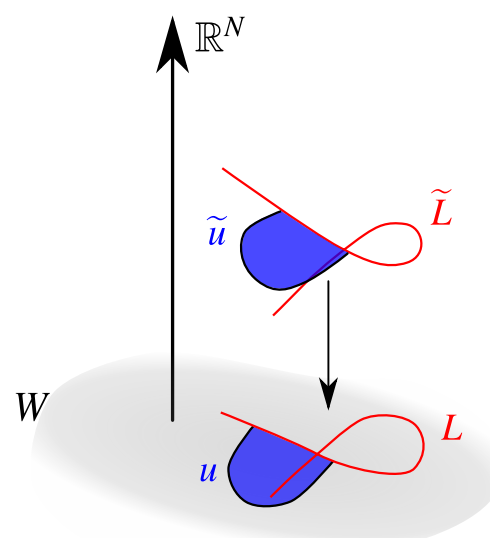

Figure 3: Eliminate the self-intersection points of $L$ by lifting all objects to $W \times \mathbb{R}^{N}$.

by setting

$$
\tilde{u}(z)= \begin{cases}(u(z), 0) & z \notin \overline{\operatorname{supp} F} \\ \left(u(z), F(z) \cdot \widetilde{\phi}\left(u^{L}(p)\right)\right) & z=(t, p) \in(-\epsilon, 0] \times \partial \Sigma .\end{cases}
$$

It is clear that the projection of $\tilde{u}$ onto the first factor gives back the original map $u$, and that the boundary of $\tilde{u}$ is mapped into $\widetilde{L}$. Similarly, we can lift any section $\sigma \in B_{u_{0}}$ to a section of $\tilde{u}_{0}^{*} T\left(W \times \mathbb{R}^{N}\right)$ by

$$
\tilde{\sigma}(z)= \begin{cases}(\sigma(z), 0) & z \notin \overline{\operatorname{supp} F} \\ \left(\sigma(z), F(z) \cdot D \tilde{\phi} \cdot \sigma\left(u_{0}^{L}(p)\right)\right) & z=(t, p) \in(-\epsilon, 0] \times \partial \Sigma .\end{cases}
$$

Note that $\tilde{\sigma}$ is tangent to $\tilde{L}$ along $\partial \Sigma$.

Choose now a Riemannian metric $g$ on $W \times \mathbb{R}^{N}$ that makes $\tilde{L}$ totally geodesic. We define a map $\operatorname{Exp}_{u_{0}}$ from the Banach space $B_{u_{0}}$ to $\mathcal{B}(\Sigma ; L)$ by associating to a section $\sigma \in B_{u_{0}}$ its lift $\tilde{\sigma}$, then applying to $\widetilde{\sigma}$ the geodesic exponential map, and projecting the resulting map back into $W$. If $\sigma$ is sufficiently small, then the image $\operatorname{Exp}_{u_{0}}(\sigma)$ will lie in $\mathcal{B}(\Sigma ; L)$, because the complement of $L_{\varnothing}$ is an open set so that for small $\sigma$ the map $\operatorname{Exp}_{u_{0}}(\sigma)$ will also intersect the complement of $L_{\varnothing}$. To see that $\operatorname{Exp}_{u_{0}}$ is injective, assume that $\operatorname{Exp}_{u_{0}}(\sigma)=\operatorname{Exp}_{u_{0}}\left(\sigma^{\prime}\right)$ for two sections $\sigma, \sigma^{\prime} \in B_{u_{0}}$. All considerations reduce to pointwise conditions for every $z \in \Sigma$. The vectors $\tilde{\sigma}(z)$ and $\tilde{\sigma}^{\prime}(z)$ lie in a subspace of $T_{\widetilde{u}_{0}(z)}\left(W \times \mathbb{R}^{N}\right)$ that is a linear graph over $T_{u_{0}(z)} W$. The geodesic exponential map of this subspace is a graph $\Gamma_{u_{0}(z)} \subset W \times \mathbb{R}^{N}$ over $W$ in a neighborhood of $u_{0}(z)$, and because $\exp (\widetilde{\sigma}(z))$ and $\exp \left(\widetilde{\sigma}^{\prime}(z)\right)$ have the same 
projection to $W$, it follows that $\tilde{\sigma}(z)=\widetilde{\sigma}^{\prime}(z)$, and since this holds for every $z \in \Sigma$, we obtain that $\sigma=\sigma^{\prime}$.

To check that $\operatorname{Exp}_{u_{0}}$ is surjective, consider a map $u \in \mathcal{B}(\Sigma ; L)$ that lies as a $W^{k, p}$-map close to $u_{0}$, and whose boundary lift $u^{L}: \Sigma \rightarrow L$ also lies close to $u_{0}^{L}$. Lift $u$ to $\tilde{u}: \Sigma \rightarrow W \times \mathbb{R}^{N}$. Then the curves $\tilde{u}_{0}$ and $\tilde{u}$ will still be close to each other, and we can project $\tilde{u}$ pointwise along $\mathbb{R}^{N}$ onto the graphs $\Gamma_{u_{0}(z)}$ spanned by the geodesic exponential maps applied to the lifts $\widetilde{\sigma}(z)$ of sections $\sigma \in B_{u_{0}}$. This way, we obtain a section $\sigma \in B_{u_{0}}$ such that $\operatorname{Exp}_{u_{0}}(\sigma)=u$.

Since we do not see locally the other intersection branches it follows that the CauchyRiemann equation defines a Fredholm operator on $\mathcal{B}(\Sigma ; L)$. For a generic adapted almost complex structure $J$, we can make sure that the space of solutions

$$
\tilde{\mathcal{M}}(\Sigma ; L)=\left\{u \in \mathcal{B}(\Sigma ; L) \mid \bar{\partial}_{J} u=0 \text {, and } u \text { is somewhere injective }\right\}
$$

is a smooth manifold (in fact, we have to use an argument as in McDuff-Salamon [19, Remark 3.2.3], because $J$ is chosen to look on one open subset of $W$ as in Proposition 17 , and on another one as in Niederkrüger [22, Section 3], but this poses no problem, because we have a precise control over the curves that intersect these model neighborhoods, and perturbing $J$ outside of these neighborhoods suffices to achieve transversality). Also, we can apply standard regularity results to see that the holomorphic curves $u \in \tilde{\mathcal{M}}(\Sigma ; L)$ are not just $W^{k, p}$-maps, but can be represented by smooth maps.

In our case, we set $\Sigma=\mathbb{D}$, and add a marked point $z_{0} \in \partial \mathbb{D}$, then we choose a path $\gamma$ that lies in the complement of $L_{\varnothing}$, and require the evaluation map at $z_{0}$ to always lie on $\gamma$. The evaluation map is smooth, and so for a generic $\gamma$, the space of such disks will be a smooth submanifold in $\tilde{\mathcal{M}}(\Sigma ; L)$ of codimension $\operatorname{dim} L-1$. The automorphism group $G:=\operatorname{Aut}\left(\mathbb{D}, z_{0}\right)$ of the disk with a marked point on its boundary is 2-dimensional, and so we obtain with the index computation in [22, Section 3.3] that $\mathcal{M}_{\gamma}:=\tilde{\mathcal{M}}(\mathbb{D} ;$ GPS $\gamma \gamma) / G$ is a 1 -dimensional manifold.

\subsection{The boundary of the GPS}

The definition of the plastikstufe in [22] requires the boundary $\partial \mathcal{P} \mathcal{S}(S)$ to be a regular leaf of the foliation. That way, $\mathcal{P S}(S)-(S \times\{0\})$ is a totally real manifold, and gives thus a Fredholm boundary condition for regarding holomorphic disks. At the same time smooth holomorphic disks in the moduli space have to be transverse to the foliation so that they cannot touch the boundary.

The compactness proof in this article relies on the assumption that once we remove a neighborhood of the core, any two leaves of the foliation on the GPS have positive 
distance. Unfortunately then in our definition of the GPS, $S \times \mathbb{D}$ is along its boundary $S \times \partial \mathbb{D}$ tangent to the contact structure. In this section, we will show by an intersection argument that there is a neighborhood of the boundary which blocks any holomorphic curve from entering it. Our definition thus implies at this point effectively the same statement as the standard one.

Proposition 16 Let $(M, \alpha)$ be a contact $(2 n+1)$-manifold, and let $F$ be a submanifold of $M$ that looks like the collar neighborhood of the boundary of a GPS, that means $F \cong S \times \mathbb{S}^{1} \times[0, \delta)$, where $S$ is a closed manifold of dimension $n-1$, and the restriction of $\alpha$ to $F$ takes the form $f(r) d \phi$ for coordinates $\left(e^{i \phi}, r\right)$ on $\mathbb{S}^{1} \times[0, \delta)$, and a function $f:[0, \delta) \rightarrow \mathbb{R}$ that vanishes for $r=0$, and is otherwise positive. Then there is a neighborhood $U$ of the boundary $\partial F:=S \times \mathbb{S}^{1} \times\{0\}$ in $M$ that is (strictly) contactomorphic to an open subset of

$$
\left(\mathbb{R} \times T^{*} S \times \mathbb{S}^{1} \times \mathbb{R}, d z+\lambda_{\text {can }}+r d \phi\right)
$$

such that $U \cap F$ lies in this model in $\{0\} \times S \times \mathbb{S}^{1} \times[0, \epsilon)$.

Proof The proof is based on a variation of the Moser trick. It is fairly standard, but for completeness we have decided to include it in Appendix A.

To describe the behavior of holomorphic curves, we have to expand the above model to include a neighborhood in the symplectization. Take the symplectic manifold

$$
\left(T^{*}\left(\mathbb{T}^{2} \times S\right) \cong T^{*} \mathbb{T}^{2} \times T^{*} S, \omega:=d\left(-p_{x} d q_{x}-p_{y} d q_{y}+\lambda_{\text {can }}\right)\right),
$$

where $\left(q_{x}, q_{y}\right)$ are the coordinates on the torus $\mathbb{T}^{2}=\mathbb{R}^{2} / \mathbb{Z}^{2}, p_{x}$ and $p_{y}$ are the directions in the cotangent bundle associated with $q_{x}$ and $q_{y}$ respectively, and $\lambda_{\text {can }}=$ $-\mathbf{p} d \mathbf{q}$ is the canonical $1-$ form on $T^{*} S$.

Choose on the manifold $S$ a Riemannian metric, and let $F$ be the function

$$
F: T^{*} S \rightarrow \mathbb{R},(\mathbf{q}, \mathbf{p}) \mapsto \frac{1}{2}\|\mathbf{p}\|^{2} .
$$

The unit cotangent bundle

$$
\left\{\left(q_{x}, q_{y}, p_{x}, p_{y} ; \mathbf{q}, \mathbf{p}\right) \in T^{*} \mathbb{T}^{2} \times T^{*} S \mid\left(p_{x}^{2}+p_{y}^{2}\right) / 2+F(\mathbf{q}, \mathbf{p})=1\right\}
$$

has a natural contact structure given as the kernel of the canonical 1 -form $\lambda=$ $-p_{x} d q_{x}-p_{y} d q_{y}+\lambda_{\text {can }}$. Its natural symplectic filling is the disk bundle in $T^{*} \mathbb{T}^{2} \times$ $T^{*} S$.

We can introduce on $T^{*} \mathbb{T}^{2} \times T^{*} S$ the almost complex structure

$$
J:=i \oplus J_{0}
$$


where $J_{0}$ is the $d \lambda_{\text {can }}$-compatible almost complex structure on $T^{*} S$ constructed in Niederkrüger [22, Appendix B], and $i$ is the standard complex structure on $T^{*} \mathbb{T}^{2}$, that is, the one defined by $i \partial_{q_{x}}=\partial_{p_{x}}$, and $i \partial_{q_{y}}=\partial_{p_{y}}$. It is easy to check that this $J$ is compatible with $\omega$, and that

$$
\lambda \circ J=p_{x} d p_{x}+p_{y} d p_{y}+\lambda_{\text {can }} \circ J_{0}=\frac{1}{2} d\left(p_{x}^{2}+p_{y}^{2}\right)+d F,
$$

where we used (see the reference above) that $d F=\lambda_{\text {can }} \circ J_{0}$.

Thus if we set $h\left(q_{x}, q_{y}, p_{x}, p_{y} ; \mathbf{q}, \mathbf{p}\right):=\left(p_{x}^{2}+p_{y}^{2}\right) / 2+F(\mathbf{q}, \mathbf{p})$, we obtain that $\lambda=-d h \circ J$. We can embed $S \times \mathbb{S}^{1} \times[0, \epsilon)$ into $T^{*} \mathbb{T}^{2} \times T^{*} S$ by using the map

$$
\begin{aligned}
S \times \mathbb{S}^{1} \times[0, \epsilon) & \rightarrow T^{*} \mathbb{T}^{2} \times T^{*} S, \\
\left(\mathbf{q}, e^{2 \pi i \phi}, r\right) & \mapsto\left(q_{x}, q_{y}, p_{x}, p_{y} ; \mathbf{q}, \mathbf{p}\right)=\left(0, \phi, \sqrt{2-r^{2}}, r ; \mathbf{q}, \mathbf{0}\right) .
\end{aligned}
$$

The image of this map lies in the contact boundary $h^{-1}(1)$, and the contact form pulls back to the required form to apply Proposition 16, so that we have found a model for a neighborhood of the boundary $\partial$ GPS in the symplectization.

We summarize these findings in the following proposition.

Proposition 17 Let $(W, \omega)$ be a strong filling of a contact manifold $(M, \alpha)$ that contains a GPS. There is a neighborhood $U$ of $\partial$ GPS in $W$ that is isomorphic to the set

$$
\begin{aligned}
W^{\prime}:=\left\{\left(q_{x}, q_{y}, p_{x}, p_{y} ; \mathbf{q}, \mathbf{p}\right) \in T^{*} \mathbb{T}^{2} \times T^{*} S \mid\right. \\
\left.\quad h\left(q_{x}, q_{y}, p_{x}, p_{y} ; \mathbf{q}, \mathbf{p}\right) \leq 1, q_{x} \in(-\epsilon, \epsilon), p_{x}>\sqrt{2-\epsilon}\right\} \subset T^{*} \mathbb{T}^{2} \times T^{*} S,
\end{aligned}
$$

for a small $\epsilon>0$ such that $U \cap$ GPS corresponds to

$$
\left\{\left(0, \phi, \sqrt{2-r^{2}}, r ; \mathbf{q}, \mathbf{0}\right) \in W^{\prime} \mid 0 \leq r<\sqrt{\epsilon}\right\} .
$$

The function $h\left(q_{x}, q_{y}, p_{x}, p_{y} ; \mathbf{q}, \mathbf{p}\right):=\left(p_{x}^{2}+p_{y}^{2}\right) / 2+F(\mathbf{q}, \mathbf{p})$ is plurisubharmonic with respect to the almost complex structure $J$ given by

$$
J=i \oplus J_{0}
$$

that is adapted to the contact manifold $(M, \alpha)$.

Proposition 18 Let $(W, \omega)$ be a strong filling of a contact manifold $(M, \alpha)$ that contains a GPS. If we choose the adapted almost complex structure $J$ described in Proposition 17 on a small neighborhood $U$ of $\partial \mathrm{GPS}$, and we extend $J$ to an adapted almost complex structure on all of $W$, then no $J$-holomorphic curve $u:(\Sigma, \partial \Sigma) \rightarrow$ 
( $W$, GPS) whose boundary components are all contractible in GPS, may intersect the neighborhood $U$.

Proof Choose for the neighborhood $U$ of $\partial$ GPS the model described in Proposition 17 together with the almost complex structure $J$ given there. This $J$ can be extended over the whole filling $(W, \omega)$. Note that the neighborhood $U$ is foliated by $J$-complex codimension 2 submanifolds of the form

$$
N\left(C_{q}, C_{p}\right):=\left\{\left(C_{q}, q_{y}, C_{p}, p_{y}\right) \times T^{*} S \mid h\left(C_{q}, q_{y}, C_{p}, p_{y} ; \mathbf{q}, \mathbf{p}\right) \leq 1\right\}
$$

for fixed values $C_{q} \in(-\epsilon, \epsilon)$ and $C_{p} \in(\sqrt{2-\epsilon}, \sqrt{2})$.

Let now $u:(\Sigma, \partial \Sigma) \rightarrow(W$, GPS $)$ be a holomorphic curve with boundary components that are all contractible in GPS, and assume that $u$ intersects the model neighborhood $U$. Write the restriction of $u$ to $V:=u^{-1}(U) \subset \Sigma$ as

$$
\left.u\right|_{V}: V \rightarrow T^{*} \mathbb{T}^{2} \times T^{*} S, z \mapsto\left(u_{q_{x}}(z), u_{q_{y}}(z), u_{p_{x}}(z), u_{p_{y}}(z) ; u_{\mathbf{q}}(z), u_{\mathbf{p}}(z)\right) .
$$

First, we will show that the coordinates $\left(q_{x}, p_{x}\right)$ are constant. If this were not the case, then the image of $\left(u_{q_{x}}, u_{p_{x}}\right)$ contains an open set, and by Sard's Theorem we find regular values $\left(C_{q}, C_{p}\right)$ with $C_{q} \neq 0$, such that $\left\{\left(u_{q_{x}}, u_{p_{x}}\right)=\left(C_{q}, C_{p}\right)\right\}$ consists of finitely many points at which the differential of $\left(u_{q_{x}}(z), u_{p_{x}}(z)\right)$ is regular, and so $N\left(C_{q}, C_{p}\right)$ has finitely many transverse intersection points with $u$. By our assumption, it is possible to cap off the holomorphic curve $u$ by adding disks that lie inside the GPS. Consider the codimension 1 submanifold (with boundary and corners)

$$
\tilde{N}\left(C_{q}, C_{p}\right):=\left\{\left(C_{q}, q_{y}, p_{x}, p_{y} ; \mathbf{q}, \mathbf{p}\right) \mid p_{x} \geq C_{p}, h\left(C_{q}, q_{y}, p_{x}, p_{y} ; \mathbf{q}, \mathbf{p}\right) \leq 1\right\} .
$$

One of its boundaries is $N\left(C_{q}, C_{p}\right)$, and because $C_{q} \neq 0$, the intersection of $\tilde{N}\left(C_{q}, C_{p}\right)$ with $M$ gives a submanifold that is disjoint from GPS, and this submanifold together with $N\left(C_{q}, C_{p}\right)$ represents the trivial homology class in $H_{2 n-2}(W)$.

The only intersections between the capped off holomorphic curve and $\partial \tilde{N}\left(C_{q}, C_{p}\right)$ lie in the subsets, where both classes are represented by $J$-holomorphic manifolds. Hence the intersection number is positive, but since $\partial \tilde{N}\left(C_{q}, C_{p}\right)$ represents the trivial homology class, this is clearly a contradiction.

It follows that $\left(u_{q_{x}}, u_{p_{x}}\right)$ have to be constant, and this in turn means that the holomorphic curve is completely contained in $N\left(u_{q_{x}}, u_{p_{x}}\right)$ which is contained in the neighborhood $U$ so that $u$ also lives in our model neighborhood $U$. In particular, it follows by the boundary condition of $u$ that $u_{q_{x}}=0$.

Project now the holomorphic curve $u: \Sigma \rightarrow T^{*} \mathbb{T}^{2} \times T^{*} S$ onto its $T^{*} S$-part to obtain a $J_{0}$-holomorphic curve in the cotangent bundle. Since the boundary of $u$ sits on 
the GPS, it follows that the boundary of the $T^{*} S$-part lies in the zero-section of $T^{*} S$, and so it has no energy, and is thus constant. So far, it follows that $u(z)$ can be written as $u(z)=\left(0, u_{q_{y}}(z), C_{p}, u_{p_{y}}(z) ; \mathbf{q}_{0}, \mathbf{0}\right)$, where $\left(u_{q_{y}}, u_{p_{y}}\right): \Sigma \rightarrow T^{*} \mathbb{S}^{1}$ is an $i$-holomorphic curve that satisfies $u_{p_{y}}(z)=+\sqrt{2-C_{p}^{2}}$ for every $z \in \partial \Sigma$. We will prove that $\left(u_{q_{y}}, u_{p_{y}}\right)$ is constant by showing that its energy is also zero. First note that the energy is given by $\int_{\Sigma} d \lambda_{\text {can }}=\int_{\partial \Sigma} \lambda_{\text {can }}$, where $\lambda_{\text {can }}$ denotes here the canonical 1 -form on $T^{*} \mathbb{S}^{1}$. Along $\partial \Sigma$ the 1 -form $\lambda_{\text {can }}=-p_{y} d q_{y}$ is identical to the closed 1 -form $-\sqrt{2-C_{p}^{2}} d q_{y}$, but since $\partial \Sigma$ represents a trivial class in homology, it follows that

$$
\int_{\partial \Sigma} \lambda_{\text {can }}=-\sqrt{2-C_{p}^{2}} \int_{\partial \Sigma} d q_{y}=0
$$

as we wanted to show, and so $\left(u_{q_{y}}, u_{p_{y}}\right)$ is also constant.

Finally, note that a $J$ chosen in such a way still gives enough freedom for achieving transversality of the Cauchy-Riemann operator: No curves are allowed to enter $U$, so we do not need to perturb $J$ in that neighborhood to achieve regularity.

\subsection{Bubbling analysis}

To obtain compactness of our moduli space, we need to distinguish two cases: Either the first derivatives of the sequence are from the beginning on uniformly bounded, and we have a subsequence with a clean limit, or otherwise if the first derivatives explode, we show that we can reparametrize the disks in a suitable way after which we do find a global uniform bound on the derivatives.

Theorem 19 Let $\Sigma$ be a Riemann surface that does not need to be compact, and may or may not have boundary. Let $\Omega_{k} \subset \Sigma$ be a family of increasing open sets that exhaust $\Sigma$, that is,

$$
\bigcup_{k} \Omega_{k}=\Sigma \quad \text { and } \quad \Omega_{k} \subset \Omega_{k^{\prime}} \text { for } k \leq k^{\prime} \text {. }
$$

Define $\partial \Omega_{k}:=\Omega_{k} \cap \partial \Sigma$. Let $(W, J)$ be a compact almost complex manifold that contains a totally real immersion $\phi: L \uparrow W$ of a compact manifold $L$, and let $u_{k}$ be a sequence of holomorphic maps $u_{k}:\left(\Omega_{k}, \partial \Omega_{k}\right) \rightarrow(W, \phi(L))$ whose derivatives are uniformly bounded on compact sets, that is, for every compact set $K \subset \Sigma$, we find a constant $C(K)>0$ such that

$$
\left\|D u_{k}(z)\right\| \leq C(K)
$$

for all $k$ and all $z \in \Omega_{k} \cap K$. Additionally assume that the restriction of $u_{k}$ to the boundary $\partial \Omega_{k}$ lifts to a collection of smooth paths $u_{k}^{L}: \partial \Omega_{k} \rightarrow L$ such that $\phi \circ u_{k}^{L}=\left.u_{k}\right|_{\partial \Omega_{k}}$. 
Then there exists a subsequence of $u_{k}$ that converges on any compact subset uniformly with all derivatives to a holomorphic curve $u_{\infty}:(\Sigma, \partial \Sigma) \rightarrow(W, \phi(L))$, whose boundary lifts to a collection of smooth paths $u_{\infty}^{L}: \partial \Sigma \rightarrow L$, and the boundary paths $u_{k}^{L}$ also converge locally uniformly to $u_{\infty}^{L}$.

Proof The theorem is well-known in case that $\partial \Sigma=\varnothing$ or that $\phi(L)$ is an embedded totally real submanifold (see for example McDuff-Salamon [19, Theorem 4.1.1]). In fact, our situation can be reduced to one, where we can apply this standard result. Using Arzelà-Ascoli it is easy to find a subsequence $u_{k}$ that converges uniformly in $C^{0}$ on any compact set to a continuous map $u_{\infty}$, and such that the lifts $u_{k}^{L}: \partial \Omega_{k} \rightarrow L$ converge in $C^{0}$ on any compact set to a lift $u_{\infty}^{L}: \partial \Sigma \rightarrow L$ with $\phi \circ u_{\infty}^{L}=\left.u_{\infty}\right|_{\partial \Sigma}$.

Let $K \subset \Sigma$ be a compact set on which we want to show uniform $C^{\infty}$-convergence. If $\partial K:=K \cap \partial \Sigma$ is empty, then the uniform convergence for the derivatives follows from the standard result. If $\partial K$ is non-empty, then cover $u_{\infty}^{L}(\partial K)$ with a finite collection of open sets $V_{1}, \ldots, V_{N}$ such that the restrictions $\left.\phi\right|_{V_{j}}$ are embeddings. We can choose slightly smaller open subsets $V_{j}^{\prime} \subset V_{j}$ for which the closure $\overline{V_{j}^{\prime}}$ is also contained in $V_{j}$, and whose union $V_{1}^{\prime} \cup \cdots \cup V_{N}^{\prime}$ still covers $u_{\infty}^{L}(\partial K)$.

Cover also $K$ itself with open sets $U_{n}$ that either do not intersect the boundary $\partial K$ or if $U_{n} \cap \partial K \neq \varnothing$, then there is a $V_{j}^{\prime}$ such that $u_{\infty}^{L}\left(U_{n} \cap \partial K\right) \subset V_{j}^{\prime}$. Only finitely many $U_{n}$ are needed to cover $K$. We get for every $U_{n} \cap K$ uniform $C^{\infty}$-convergence, because now if $U_{n}$ intersects $\partial K$ we can use the standard result: For $k$ large enough $u_{k}\left(U_{n} \cap \partial K \cap \Omega_{k}\right)$ will be contained in the larger subset $V_{j}$, on which $\phi$ is an embedding.

\section{Theorem 20 (Gromov compactness) Let}

$$
u_{k}:(\mathbb{D}, \partial \mathbb{D}) \rightarrow\left(W, \mathrm{GPS}^{*}\right)
$$

be a sequence of holomorphic disks that represent elements in the moduli space $\mathcal{M}_{\gamma}$. There exists a family $\phi_{k}: \mathbb{D} \rightarrow \mathbb{D}$ of biholomorphisms such that $u_{k} \circ \phi_{k}$ contains a subsequence converging uniformly in $C^{\infty}$ to a holomorphic disk

$$
u_{\infty}:(\mathbb{D}, \partial \mathbb{D}) \rightarrow\left(W, \mathrm{GPS}^{*}\right)
$$

that represents again an element in $\mathcal{M}_{\gamma}$. Note in particular that the boundary of $u_{\infty}$ also lies in the interior of the GPS.

Proof Choose the metric on $W$ that is compatible with both $J$ and $\omega$, and endow the disk $\mathbb{D} \subset \mathbb{C}$ with the restriction of the standard metric $g_{0}$ on the complex plane. Denote by $\left\|D u_{k}(z)\right\|_{\mathbb{D}}$ the norm of the differential of $u_{k}$ at a point $z \in \mathbb{D}$ with respect 
to $g_{0}$ on the disk, and the chosen metric on $W$. If $\left\|D u_{k}\right\|_{\mathbb{D}}$ is uniformly bounded for all $k \in \mathbb{N}$ and all $z \in \mathbb{D}$, then by Theorem 19 we are done.

So assume this to be false, then there exists (by going to a subsequence if necessary) a sequence $z_{k} \in \mathbb{D}$ such that

$$
\left\|D u_{k}\left(z_{k}\right)\right\|_{\mathbb{D}} \rightarrow \infty,
$$

and in fact by rotating the disk, we may assume that all $z_{k}$ lie on the ray $[0,1]$.

Let $\mathbb{H} \subset \mathbb{C}$ be the upper half plane $\{z \in \mathbb{C} \mid \operatorname{Im} z \geq 0\}$ endowed with the Euclidean metric, and denote by $\|D v(z)\|_{\mathbb{H}}$ the norm of the differential of a map $v: \mathbb{H} \rightarrow W$ at a point $z \in \mathbb{H}$ with respect to the Euclidean metric on the half plane, and the chosen metric on $W$. We can map the half plane into the unit disk using the biholomorphism

$$
\Phi: \mathbb{H} \rightarrow \mathbb{D}-\{-1\}, z \mapsto \frac{i-z}{i+z},
$$

and use this to pull-back the sequence of disks to $u_{k}^{\mathbb{H}}:=u_{k} \circ \Phi:(\mathbb{H}, \mathbb{R}) \rightarrow(W$, GPS $)$. Introduce also the notation $z_{k}^{\mathbb{H}}:=\Phi^{-1}\left(z_{k}\right), \mathbb{D}\left(z_{0}\right):=\left\{z \in \mathbb{C}|| z-z_{0} \mid<1\right\}$, and $\mathbb{D}_{r}\left(z_{0}\right):=\left\{z \in \mathbb{C}|| z-z_{0} \mid<r\right\}$. The map $\Phi$ is not an isometry, but on compact sets of the upper half plane, $\Phi^{*} g_{0}$ is equivalent to the Euclidean metric. Hence it follows that also the sequence $\left\|D u_{k}^{\mathbb{H}}\left(z_{k}^{\mathbb{H}}\right)\right\|_{\mathbb{H}}$ cannot be bounded.

Apply the Hofer Lemma (see for example [19, Lemma 4.6.4] and Figure 4) for fixed $k$, and $\delta=1 / 2$, that means, restrict $\left\|D u_{k}^{\mathbb{H}}\right\|_{\mathbb{H}}$ to the unit disk $\mathbb{D}\left(z_{k}^{\mathbb{H}}\right) \cap \mathbb{H}$. There is a positive $\epsilon_{k}$ with $\epsilon_{k} \leq 1 / 2$, and a $y_{k} \in \mathbb{D}_{1 / 2}\left(z_{k}^{\mathbb{H}}\right) \cap \mathbb{H}$ such that

$$
\left\|D u_{k}^{\mathbb{H}}\left(z_{k}^{\mathbb{H}}\right)\right\|_{\mathbb{H}} \leq 2 \epsilon_{k}\left\|D u_{k}^{\mathbb{H}}\left(y_{k}\right)\right\|_{\mathbb{H}} \quad \text { and } \quad\left\|D u_{k}^{\mathbb{H I}}(z)\right\|_{\mathbb{H}} \leq 2\left\|D u_{k}^{\mathbb{H I}}\left(y_{k}\right)\right\|_{\mathbb{H}}
$$

for all $z \in \mathbb{D}_{\epsilon_{k}}\left(y_{k}\right) \cap \mathbb{H}$.

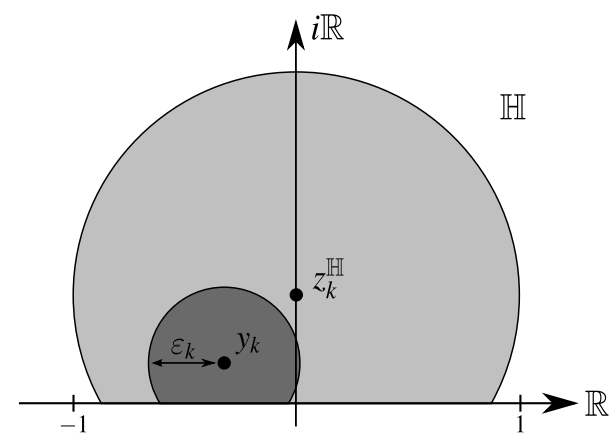

Figure 4: Using the Hofer Lemma we find in the unit disk around $z_{k}^{\mathbb{H I}}$ a smaller domain $\mathbb{D}_{\epsilon_{k}}\left(y_{k}\right)$ that can be rescaled to recover a bubble. 
Set $c_{k}:=\left\|D u_{k}^{\mathbb{H}}\left(y_{k}\right)\right\|_{\mathbb{H}}$. First we will show that for large $k$, all the disks $\mathbb{D}_{\epsilon_{k}}\left(y_{k}\right)$ intersect the boundary $\partial \mathbb{H}=\mathbb{R}$ of the half plane. Even stricter, there exists a constant $K>0$ such that $c_{k} \operatorname{Im}\left(y_{k}\right)<K$ for all $k$ (if the disks intersect the real line, we have $\operatorname{Im} y_{k}<\epsilon_{k}$, multiplying with $c_{k}$ on both sides would still allow the left side to be unbounded). Suppose that such a constant did not exist, so that by going to a subsequence, $c_{k} \operatorname{Im} y_{k}$ converges monotonously to $\infty$. Define $H_{k}:=\{z \in \mathbb{C} \mid \operatorname{Im} z \geq$ $\left.-c_{k} \operatorname{Im} y_{k}\right\}$, and a sequence of biholomorphisms

$$
\phi_{k}: \mathbb{D}_{\epsilon_{k} c_{k}} \cap H_{k} \rightarrow \mathbb{D}_{\epsilon_{k}}\left(y_{k}\right) \cap \mathbb{H}, z \mapsto \frac{z}{c_{k}}+y_{k} .
$$

Pulling back, we find holomorphic maps $\widehat{u}_{k}:=u_{k}^{\mathbb{H}} \circ \phi_{k}: \mathbb{D}_{\epsilon_{k} c_{k}} \cap H_{k} \rightarrow W$ with $\left\|D \widehat{u}_{k}(0)\right\|=1$, and $\left\|D \widehat{u}_{k}\right\| \leq 2$ everywhere else. Using Theorem 19 (or just for example [19, Theorem 4.1.1]), proves that there exists a subsequence that converges locally uniformly with all derivatives to a non-constant map $\widehat{u}_{\infty}: \mathbb{C} \rightarrow W$. The standard removal of singularity theorem yields then a non-constant holomorphic sphere, but this is a contradiction, because in a symplectically aspherical manifold its energy would have to be zero. Thus there is a constant $K>0$ such that $c_{k} \operatorname{Im}\left(y_{k}\right)<K$.

Now we slightly modify the charts used above to achieve that the reparametrized domains lie inside the upper half plane, and that their boundary lies in the real line. Set $y_{k}^{\prime}:=c_{k} \operatorname{Im} y_{k}$ and $r_{k}:=\epsilon_{k} c_{k}$, and consider the following sequence of biholomorphisms

$$
\psi_{k}: \mathbb{D}_{r_{k}}\left(i y_{k}^{\prime}\right) \cap \mathbb{H} \rightarrow \mathbb{D}_{\epsilon_{k}}\left(y_{k}\right) \cap \mathbb{H}, z \mapsto \frac{z}{c_{k}}+\operatorname{Re} y_{k} .
$$

Note that the intersection of $\mathbb{D}_{\epsilon_{k}}\left(y_{k}\right)$ with the real line is given by the interval

$$
\mathbb{D}_{\epsilon_{k}}\left(y_{k}\right) \cap \mathbb{R} \subset \mathbb{D}\left(z_{k}^{\mathbb{H}}\right) \cap \mathbb{R} \subset(-1,1) .
$$

The image of the interval $(-1,1)$ under $\Phi$ is the segment on the boundary of the unit disk enclosed between the angles $(-\pi / 2, \pi / 2)$. This means that the boundary part of the disk that is affected by the reparametrization lies on the right half of the complex plane.

On the domain of the reparametrized maps $\hat{u}_{k}:=u_{k}^{\mathbb{H}} \circ \psi_{k}: \mathbb{D}_{r_{k}}\left(i y_{k}^{\prime}\right) \cap \mathbb{H} \rightarrow W$ we have $\left\|D \widehat{u}_{k}\right\| \leq 2$, and $\left\|D \widehat{u}_{k}\left(i y_{k}^{\prime}\right)\right\|=1$. We can also find a subsequence of $\widehat{u}_{k}$ with increasing domains, that is, $\mathbb{D}_{r_{k}}\left(i y_{k}^{\prime}\right) \subset \mathbb{D}_{r_{l}}\left(i y_{l}^{\prime}\right)$ for all $l \geq k$, by using that the $y_{k}^{\prime}$ are all bounded while the radii of the disks $r_{k}$ become arbitrarily large. Then Theorem 19 provides a subsequence of the $\hat{u}_{k}$ that converges locally uniformly with all derivatives to a holomorphic map $\hat{u}_{\infty}:(\mathbb{H}, \mathbb{R}) \rightarrow(W, \mathrm{GPS})$. To see that $\hat{u}_{\infty}$ is not constant, take a subsequence such that $y_{k}^{\prime}$ converges to $y_{\infty}^{\prime}$. The norm of the derivative of $u_{\infty}$ at $i y_{\infty}$ is $\left\|D \widehat{u}_{\infty}\left(i y_{\infty}^{\prime}\right)\right\|=1$, because $\left\|D \widehat{u}_{\infty}\left(i y_{\infty}^{\prime}\right)-D \hat{u}_{k}\left(i y_{k}^{\prime}\right)\right\| \leq$ 
$\left\|D \widehat{u}_{\infty}\left(i y_{\infty}^{\prime}\right)-D \widehat{u}_{\infty}\left(i y_{k}^{\prime}\right)\right\|+\left\|D \widehat{u}_{\infty}\left(i y_{k}^{\prime}\right)-D \widehat{u}_{k}\left(i y_{k}^{\prime}\right)\right\|$ becomes arbitrarily small. The first term is small, because the differential of $\hat{u}_{\infty}$ is continuous, the second can be estimated by using that the convergence of $\widehat{u}_{k}$ to $\widehat{u}_{\infty}$ is uniform on a small compact neighborhood of $i y_{\infty}^{\prime}$.

Let us come back to the initial family of disks $u_{k}:(\underset{\sim}{\mathbb{D}}, \partial \mathbb{D}) \rightarrow(W$, GPS $)$. The maps $\psi_{k}$ induce reparametrizations of the whole disk by $\tilde{\psi}_{k}:=\Phi \circ \psi_{k} \circ \Phi^{-1}$. The image of a compact subset of $\mathbb{D}-\{-1\}$ under $\Phi^{-1}$ is a compact subset in $\mathbb{H}$, so that we get on any compact subset of $\mathbb{D}-\{-1\}$ uniform $C^{\infty}$-convergence of $u_{k} \circ \widetilde{\psi}_{k}$ to $u_{\infty}:=\widehat{u}_{\infty} \circ \Phi^{-1}$. To complete the proof of our compactness theorem, we have to show that the first derivatives of $u_{k} \circ \widetilde{\psi}_{k}$ are also uniformly bounded in a neighborhood of $\{-1\}$. We will do this by showing that if the derivatives were not bounded, then the curves $u_{k} \circ \widetilde{\psi}_{k}$ would intersect many leaves of the foliation on the GPS twice.

Recall that there is a continuous map $\theta:$ GPS* $\rightarrow \mathbb{S}^{1}$ that labels the leaves of the foliation on GPS* bijectively. The holomorphic curve

$$
(\mathbb{H}-\{0\},(-\infty, 0) \cup(0, \infty)) \rightarrow(W, \text { GPS }), \quad z \mapsto u_{\infty}(-\Phi(z))
$$

has finite energy, and because it intersects every leaf of the foliation of GPS* at most once (the maps $\left.\theta \circ u_{k} \circ \widetilde{\psi}_{k}\right|_{\partial \mathbb{D}}$ are all bijective), we can apply the removal of singularity theorem in the form described in Theorem 21 . The consequence for $u_{\infty}$ is that the composition $\left.\theta \circ u_{\infty}\right|_{\partial \mathbb{D}-\{-1\}}$ extends to a continuous map $\mathbb{S}^{1} \rightarrow \mathbb{S}^{1}$ that is strictly monotonous. In fact, $\left.\theta \circ u_{\infty}\right|_{\partial \mathbb{D}-\{-1\}}$ covers the whole circle with exception of the point

$$
e^{i \phi_{\infty}}:=\lim _{\phi \rightarrow \pm \pi} \theta \circ u_{\infty}\left(e^{i \phi}\right)
$$

and so for any $\epsilon$-neighborhood $U_{\epsilon} \subset \mathbb{S}^{1}$ of $e^{i \phi_{\infty}}$, we find a $\delta>0$ such that $\left\{\theta \circ u_{k} \circ\right.$ $\left.\tilde{\psi}_{k}\left(e^{i \phi}\right) \mid \phi \in(-\pi+\delta, \pi-\delta)\right\}$ covers for any sufficiently large $k$ the complement $\mathbb{S}^{1}-U_{\epsilon}$ of $U_{\epsilon}$.

Let $K_{+}$be the arc $\left\{e^{i \phi} \mid \phi \in(-\pi / 2, \pi / 2)\right\}$, that is, the right half of the unit circle. Remember that the $\widetilde{\psi}_{k}\left(K_{+}\right)$exhaust $\partial \mathbb{D}-\{-1\}$, and so it follows in particular that because the derivatives $\left\|D u_{k}\right\|_{\mathbb{D}}$ were not uniformly bounded on the ray $[0,1] \subset \mathbb{D}$, the original unparametrized disks $u_{k}:(\mathbb{D}, \partial \mathbb{D}) \rightarrow(W, \mathrm{GPS})$ intersect on $K_{+}$for sufficiently large $k$ almost all leaves of the foliation of the GPS.

Assume now that the first derivatives of the reparametrized disks $u_{k} \circ \widetilde{\psi}_{k}$ are not uniformly bounded in a neighborhood of $\{-1\}$. By the same reasoning as above, it follows that the $u_{k} \circ \widetilde{\psi}_{k}$ intersect almost all leaves of the GPS on the segment $K_{-}=\left\{e^{i \phi} \mid \phi \in(\pi / 2,3 \pi / 2)\right\}$, that is, on the left half of the circle. With "almost all", 
we mean that we find an $e^{i \phi_{1}} \in \mathbb{S}^{1}$ such that for any neighborhood $U \subset \mathbb{S}^{1}$ of $e^{i \phi_{1}}$ there exists a $k_{U}>0$ for which all $\theta \circ u_{k} \circ \widetilde{\psi}_{k}\left(K_{-}\right)$with $k>k_{U}$ cover $\mathbb{S}^{1}-U$.

This leads to a contradiction, because if the segments $\theta \circ u_{k} \circ \tilde{\psi}_{k}\left(K_{-}\right)$intersect almost all leaves of the foliation, it follows that in the limit almost no leaves will be intersected by $\theta \circ u_{k} \circ \widetilde{\psi}_{k}\left(K_{+}\right)$. But we know that $\left.\theta \circ u_{\infty}\right|_{\mathbb{S} 1}$ is not constant in a neighborhood of 1 (because $u_{\infty}$ is not constant), and so because of the local uniform convergence, we find an open set $U \subset \mathbb{S}^{1}$ that is contained in the images $\theta \circ u_{k} \circ \widetilde{\psi}_{k}\left(K_{+}\right)$for any $k$ that is sufficiently large.

In our special situation, we only need the following very weak form of removal of singularity, which states that the punctured boundary of a holomorphic curve approaches from both sides of the puncture the same leaf of the foliation of the GPS.

Theorem 21 (Removal of singularity) Let $(W, \omega)$ be a compact symplectic manifold with convex boundary $\partial W=(M, \alpha)$. Assume that $M$ contains a GPS $S \times \mathbb{D} \rightarrow M$, and choose an adapted almost complex structure $J$ on $W$. Let

$$
u:\left(\mathbb{D}_{\epsilon} \cap \mathbb{H}-\{0\},(-\epsilon, 0) \cup(0, \epsilon)\right) \rightarrow\left(W, \mathrm{GPS}^{*}\right)
$$

be a non-constant holomorphic curve that has finite energy, and let $\theta$ : GPS $\rightarrow \mathbb{S}^{1}$ be the continuous map that labels bijectively the leaves of the foliation on GPS* Assume additionally that $u$ intersects every leaf at most a finite number of times.

Then we find a continuous path $\widehat{c}:(-\epsilon, \epsilon) \rightarrow \mathbb{S}^{1}$ with

$$
\left.\widehat{c}\right|_{(-\epsilon, 0) \cup(0, \epsilon)}=\left.\theta \circ u\right|_{(-\epsilon, 0) \cup(0, \epsilon)} .
$$

A more geometric way to state this result is to say that the boundary segments of the holomorphic curve approach the same leaf from both sides of the origin asymptotically.

Proof Denote the segments composing the map $\left.\theta \circ u\right|_{(-\epsilon, 0) \cup(0, \epsilon)}$ by $c_{-}:(-\epsilon, 0) \rightarrow$ $\mathbb{S}^{1}$, and $c_{+}:(0, \epsilon) \rightarrow \mathbb{S}^{1}$. By Corollary 8 , both maps $c_{ \pm}$are strictly increasing, and from our assumption it follows that the $c_{ \pm}$are bounded close to 0 (in the sense that they do not turn infinitely often as $z \rightarrow 0$ ), because $u$ intersects every leaf only a finite number of times, so that we find continuous extensions $\hat{c}_{-}:(-\epsilon, 0] \rightarrow \mathbb{S}^{1}$, and $\widehat{c}_{+}:[0, \epsilon) \rightarrow \mathbb{S}^{1}$. Assume for the limits $\widehat{c}_{-}(0) \neq \widehat{c}_{+}(0)$, contrary to what we want to show. 
One of the basic ingredients in all proofs of this type is the following estimate for the energy of $u$

$$
\begin{aligned}
E(u)=\int_{\mathbb{D}_{\epsilon} \cap \mathbb{H}-\{0\}} u^{*} \omega & =\int_{0}^{\epsilon} \int_{0}^{\pi} \frac{\left|\partial_{\phi} u\left(r e^{i \phi}\right)\right|^{2}}{r^{2}} r d r \wedge d \phi \\
& \geq \int_{0}^{\epsilon}\left(\int_{0}^{\pi}\left|\partial_{\phi} u\left(r e^{i \phi}\right)\right| d \phi\right)^{2} \frac{d r}{\pi r}=\int_{0}^{\epsilon} \frac{L\left(\gamma_{r}\right)^{2}}{\pi r} d r,
\end{aligned}
$$

where $\gamma_{r}$ is the image $\left\{u\left(r e^{i \phi}\right) \mid \phi \in[0, \pi]\right\}$ of the half-circle of radius $r$ in the hyperbolic plane, and $L\left(\gamma_{r}\right)$ is its length with respect to the compatible metric on $W$. It is clear that $L\left(\gamma_{r}\right)$ cannot be bounded from below, because the energy $E(u)$ is finite. Choose a small $\delta>0$, such that the $\delta$-neighborhoods $U_{-}, U_{+} \subset \mathbb{S}^{1}$ around $\hat{c}_{-}(0)$ and $\widehat{c}_{+}(0)$ respectively do not overlap. Then there is an $\epsilon^{\prime}>0$ for which the segment $\left[0, \epsilon^{\prime}\right)$ is contained in $\widehat{c}_{+}^{-1}\left(U_{+}\right)$, and $\left(-\epsilon^{\prime}, 0\right]$ is contained in $\widehat{c}_{-}^{-1}\left(U_{-}\right)$, and by the geometry of the foliation on the GPS, all the points in $u\left(\left(0, \epsilon^{\prime}\right)\right)$ are at distance more than $C>0$ from the points in $u\left(\left(-\epsilon^{\prime}, 0\right)\right)$. In particular it follows that the length $L\left(\gamma_{r}\right)$ for any $r \in\left(0, \epsilon^{\prime}\right)$ is larger than $C$, and so by the energy inequality given above, we get a contradiction to $\widehat{c}_{-}(0) \neq \widehat{c}_{+}(0)$.

\section{Outlook and open questions}

\subsection{Definition of a capacity invariant for contact manifolds}

One obvious application of the observations made in this paper is the definition of a capacity invariant for higher dimensional contact manifolds. Unfortunately, we were not able to measure the "size" efficiently in a numerical way so that our invariant is rather rough.

To measure the capacity, we choose a contact manifold $\left(N, \xi_{N}\right)$ that will serve as the "testing probe". Then we can define for any contact manifold $(M, \xi)$ with $\operatorname{dim} M=$ $2 k+\operatorname{dim} N$, and $k \geq 1$, an invariant $C_{\xi_{N}}$ defined as follows:

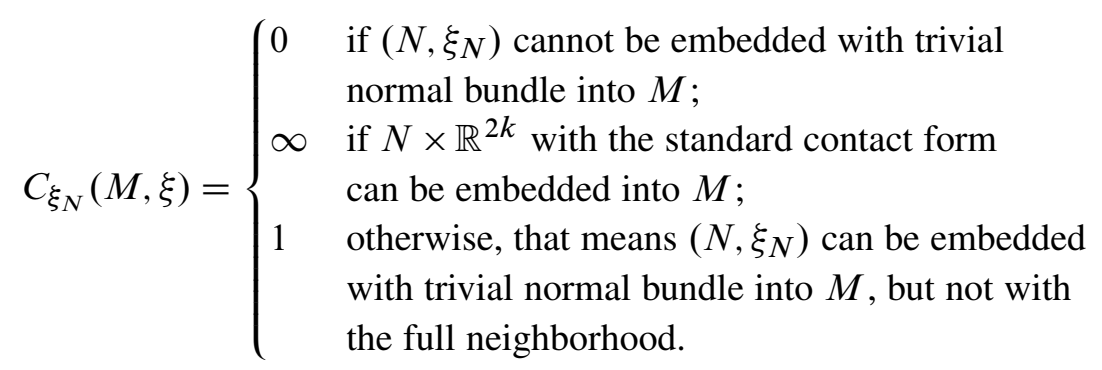


This way, we obtain for the standard sphere $\left(\mathbb{S}^{2 n-1}, \xi_{0}\right)$ that $C_{\xi_{0}}(M, \xi)=\infty$ for any contact manifold $(M, \xi)$, see Example 3(a). If $\left(N, \xi_{-}\right)$is an overtwisted contact 3 -manifold, and if $(M, \xi)$ is a manifold with exact symplectic filling, then it follows from Corollary 13 that $C_{\xi_{-}}(M, \xi)<\infty$.

The most important problem in this context would be to find examples of contact manifolds that do allow the embedding of an overtwisted contact manifold $N$ with the full model neighborhood $N \times \mathbb{R}^{2 k}$, because otherwise it is so far unclear whether the capacity $C_{\xi_{N}}$ is able to distinguish any manifolds at all. Possible candidates to check are the following:

Question 22 Let $(M, \alpha)$ be a closed contact manifold. F Bourgeois described in [2] a construction of a contact structure on $M \times \mathbb{T}^{2}$ for which every fiber $M \times\{p\}$ with $p \in \mathbb{T}^{2}$ is contactomorphic to the initial manifold. How large is the tubular neighborhood of such a fiber?

Question 23 E Giroux conjectures that contact manifolds of arbitrary dimension obtained from the negative stabilization of an open book should be "overtwisted". The simplest example of such a manifold is a sphere $\left(\mathbb{S}^{2 n-1}, \alpha_{-}\right)$constructed by taking the cotangent bundle $T^{*} \mathbb{S}^{n-1}$ for the pages, and a negative Dehn-Seidel twist as the monodromy map (see Example 5(a)). How large is the tubular neighborhood of $\left(\mathbb{S}^{3}, \alpha_{-}\right)$in a higher dimensional sphere?

\subsection{Contact structures on $\mathbb{R}^{2 n+1}$}

In Example 4 it was shown that a sufficiently large neighborhood of $\mathbb{R}^{3} \times\{\boldsymbol{0}\}$ in $\left(\mathbb{R}^{3} \times \mathbb{C}^{k}, \alpha_{-}+\sum_{j=1}^{k} r_{j}^{2} d \theta_{j}\right)$ and a sufficiently small one are not contactomorphic because small neighborhoods can be embedded into Darboux charts.

Question 24 Are all sufficiently small contractible neighborhoods of $\mathbb{R}^{3} \times\{\mathbf{0}\}$ in $\mathbb{R}^{3} \times \mathbb{C}^{k}$ contactomorphic to each other. In particular it would be interesting to see whether these spaces are contactomorphic to $\left(\mathbb{R}^{2 k+3}, \xi_{0}\right)$, because otherwise it would mean that $\mathbb{R}^{2 k+3}$ contains contractible subsets with exotic contact structures.

\subsection{Wall's embedding theorem for contact manifolds}

By a theorem of C T C Wall, every closed 3-manifold can be embedded into $\mathbb{S}^{5}$. In view of Remark 2, the following question would be interesting:

Question 25 Is it possible to embed every closed contact 3-manifold by a contactomorphism into the standard 5-sphere? If not, an intriguing problem would be to find obstructions. 


\section{Appendix A Proof of Proposition 16}

Proposition 16 Let $(M, \alpha)$ be a contact $(2 n+1)$-manifold, and let $F$ be a submanifold of $M$ that looks like the collar neighborhood of the boundary of a GPS, that means $F \cong S \times \mathbb{S}^{1} \times[0, \delta)$, where $S$ is a closed manifold of dimension $n-1$, and the restriction of $\alpha$ to $F$ takes the form $f(r) d \phi$ for coordinates $\left(e^{i \phi}, r\right)$ on $\mathbb{S}^{1} \times[0, \delta)$, and a function $f:[0, \delta) \rightarrow \mathbb{R}$ that vanishes for $r=0$, and is otherwise positive. Then there is a neighborhood $U$ of the boundary $\partial F:=S \times \mathbb{S}^{1} \times\{0\}$ in $M$ that is (strictly) contactomorphic to an open subset of

$$
\left(\mathbb{R} \times T^{*} S \times \mathbb{S}^{1} \times \mathbb{R}, d z+\lambda_{\text {can }}+r d \phi\right)
$$

such that $U \cap F$ lies in this model in $\{0\} \times S \times \mathbb{S}^{1} \times[0, \epsilon)$.

Proof The restriction of the contact form to $F$ can be written as

$$
\left.\alpha\right|_{T F}=f d \phi
$$

with a smooth non-negative function $f$ that only depends on the coordinate of $[0, \delta)$, and only vanishes on the boundary $\partial F:=S \times \mathbb{S}^{1} \times\{0\}$. The 2 -form $d \alpha$ is a symplectic form on the $(2 n)$-dimensional kernel $\xi=\operatorname{ker} \alpha$, so in particular $\left.d \alpha\right|_{T F}$ cannot vanish at any point $p \in \partial F$ of the boundary, because otherwise $T_{p} F$ would be an $(n+1)-$ dimensional isotropic subspace of $\left(\xi_{p}, d \alpha\right)$. It follows that the radial derivative of $f$ has to be positive on $\partial F$, and so we can find a collar neighborhood of the boundary $\partial F$ diffeomorphic to $S \times \mathbb{S}^{1} \times[0, \epsilon)$ with coordinates $\left(s, e^{i \phi}, r\right)$ such that $f\left(s, e^{i \phi}, r\right)=r$, by using the flow of the vector field $X=\nabla f /\|\nabla f\|^{2}$ for a gradient $\nabla f$ defined with respect to any Riemannian metric $g$. If $g$ is chosen to be $\phi$-invariant, then $\alpha$ restricts on this collar neighborhood to $r d \phi$.

Consider now the normal bundle of $F$ in $M$. A trivialization can be obtained by realizing first that the Reeb field $X_{\text {Reeb }}$ is transverse to a collar neighborhood of $\partial F$, because $\left.T F\right|_{\partial F}$ lies in the contact structure, so that there is a small neighborhood, where $X_{\text {Reeb }}$ is transverse to $F$. Choose now an almost complex structure $J$ on $\xi=\operatorname{ker} \alpha$ that is compatible with $d \alpha$ such that $J$ leaves the subbundle over $\partial F$ spanned by $\left\langle\partial_{r}, \partial_{\phi}\right\rangle$ invariant. The submanifolds $S_{\left(e^{i \phi}, r\right)}:=S \times\left\{\left(e^{i \phi}, r\right)\right\}$, with $\left(e^{i \phi}, r\right)$ fixed, are all tangent to the contact structure, and it follows that $J \cdot T S_{\left(e^{i \phi}, r\right)}$ is transverse to $F$, because if there were a direction $X \in T S$, such that $J X \in T F$, then

$$
0<d \alpha(X, J X)=d r \wedge d \phi(X, J X)=0 .
$$

The normal bundle of the collar is isomorphic to the sum of the trivial bundle spanned by the Reeb field, and the bundle $J \cdot T S_{\left(e^{i \phi}, r\right)}$. With the tubular neighborhood theorem 
it follows that there is an open set around $\partial F$ diffeomorphic to $\mathbb{R} \times T^{*} S \times \mathbb{S}^{1} \times(-\epsilon, \epsilon)$, and the collar neighborhood of $\partial F$ lies in $\{0\} \times S \times \mathbb{S}^{1} \times[0, \epsilon)$.

In the final step, we use a version of the Moser trick explained for example by Geiges [12, Theorem 2.24] to find a vector field $X_{t}$ that isotopes the given contact form $\alpha_{0}=\alpha$ into the desired one $\alpha_{1}=d z+\lambda_{\text {can }}+r d \phi$. Let $\alpha_{t}, t \in[0,1]$, be the linear interpolation between both 1-forms. Note that the 1-forms $\alpha_{0}$ and $\alpha_{1}$, and the 2 -forms $d \alpha_{0}$ and $d \alpha_{1}$ are identical along $F$, so that all $\alpha_{t}$ are contact forms if we restrict to a sufficiently small neighborhood of $\partial F$. We wish to construct an isotopy $\psi_{t}$ defined around $\partial F$ such that $\psi_{t}^{*} \alpha_{t}=\alpha_{0}$. The field $X_{t}$ generating such an isotopy has to satisfy the equation

$$
\mathcal{L}_{X_{t}} \alpha_{t}+\dot{\alpha}_{t}=0 \text {. }
$$

By writing $X_{t}=H_{t} R_{t}+Y_{t}$, where $H_{t}$ is a smooth function, $R_{t}$ is the Reeb vector field of $\alpha_{t}$, and $Y_{t} \in \operatorname{ker} \alpha_{t}$, we obtain plugging then $R_{t}$ into the equation above

$$
d H_{t}\left(R_{t}\right)=-\dot{\alpha}_{t}\left(R_{t}\right) .
$$

The vector field $Y_{t}$ is completely determined by $H_{t}$, because $Y_{t}$ satisfies the equations

$$
\begin{aligned}
\iota_{Y_{t}} \alpha_{t} & =0, \\
\iota_{Y_{t}} d \alpha_{t} & =-d H_{t}-\dot{\alpha}_{t},
\end{aligned}
$$

hence it suffices to find a suitable function $H_{t}$. Consider the 1-parameter family of Reeb fields $R_{t}$ as a single vector field on the manifold $[0,1] \times\left(\mathbb{R} \times T^{*} S \times \mathbb{S}^{1} \times \mathbb{R}\right)$. Since $R_{t}$ is just $\partial_{z}$ along $[0,1] \times S \times \mathbb{S}^{1} \times[0, \epsilon)$, it is transverse to the submanifold $N:=[0,1] \times\left(\{0\} \times T^{*} S \times \mathbb{S}^{1} \times \mathbb{R}\right)$ (on a small neighborhood of $[0,1] \times \partial F$ in $[0,1] \times F)$, and it is possible to define a solution $H_{t}$ to $d H_{t}\left(R_{t}\right)=-\dot{\alpha}_{t}\left(R_{t}\right)$, such that $\left.H_{t}\right|_{N} \equiv 0$. In fact, because $\left.\dot{\alpha}_{t}\right|_{F}=0$, it follows that $\left.d H_{t}\right|_{F}=0$, and so the vector field $X_{t}=H_{t} R_{t}+Y_{t}$ vanishes on the collar of $\partial F$. Hence $X_{t}$ can be integrated on a small neighborhood of the collar $\partial F \times[0, \epsilon)$ up to time 1 , and the collar itself is not moved under the flow, which finishes the proof of the proposition.

\section{References}

[1] L Bates, G Peschke, A remarkable symplectic structure, J. Differential Geom. 32 (1990) 533-538 MR1072917

[2] F Bourgeois, Odd dimensional tori are contact manifolds, Int. Math. Res. Not. (2002) 1571-1574 MR1912277

[3] F Bourgeois, O van Koert, Contact homology of left-handed stabilizations and plumbing of open books arXiv:0803.0391 To appear in Contemp. Math. 
[4] Y Chekanov, O van Koert, F Schlenk, Minimal atlases of closed contact manifolds, from: "New perspectives and challenges in symplectic field theory", CRM Proc. Lecture Notes 49, Amer. Math. Soc. (2009) 73-112 MR2555934

[5] Y Eliashberg, Three lectures on symplectic topology in Cala Gonone. Basic notions, problems and some methods, Rend. Sem. Fac. Sci. Univ. Cagliari 58 (1988) 27-49 MR1122856 Conference on Differential Geometry and Topology (Sardinia, 1988)

[6] Y Eliashberg, New invariants of open symplectic and contact manifolds, J. Amer. Math. Soc. 4 (1991) 513-520 MR1102580

[7] Y Eliashberg, Classification of contact structures on $\mathbb{R}^{3}$, Internat. Math. Res. Notices (1993) 87-91 MR1208828

[8] Y Eliashberg, S S Kim, L Polterovich, Geometry of contact transformations and domains: orderability versus squeezing, Geom. Topol. 10 (2006) 1635-1747 MR2284048

[9] Y Eliashberg, N Mishachev, Introduction to the h-principle, Graduate Studies in Mathematics 48, American Mathematical Society (2002) MR1909245

[10] J B Etnyre, Planar open book decompositions and contact structures, Int. Math. Res. Not. (2004) 4255-4267 MR2126827

[11] J B Etnyre, K Honda, Cabling and transverse simplicity, Ann. of Math. (2) 162 (2005) 1305-1333 MR2179731

[12] H Geiges, Contact geometry, from: "Handbook of differential geometry Vol II", Elsevier/North-Holland, Amsterdam (2006) 315-382 MR2194671

[13] H Geiges, An introduction to contact topology, Cambridge Studies in Advanced Mathematics 109, Cambridge University Press (2008) MR2397738

[14] D Gilbarg, N S Trudinger, Elliptic partial differential equations of second order, Classics in Mathematics, Springer, Berlin (2001) MR1814364 Reprint of the 1998 edition

[15] M Gromov, Pseudoholomorphic curves in symplectic manifolds, Invent. Math. 82 (1985) 307-347 MR809718

[16] M Gromov, Partial differential relations, Ergebnisse der Mathematik und ihrer Grenzgebiete (3) 9, Springer, Berlin (1986) MR864505

[17] S Ivashkovich, V Shevchishin, Reflection principle and J-complex curves with boundary on totally real immersions, Commun. Contemp. Math. 4 (2002) 65-106 MR1890078

[18] E Lerman, Contact fiber bundles, J. Geom. Phys. 49 (2004) 52-66 MR2077244

[19] D McDuff, D Salamon, J-holomorphic curves and symplectic topology, American Mathematical Society Colloquium Publications 52, American Mathematical Society (2004) MR2045629 
[20] A Mori, Global models of contact forms, J. Math. Sci. Univ. Tokyo 11 (2004) 447-454 MR2110923

[21] M-P Muller, Une structure symplectique sur $\mathbb{R}^{6}$ avec une sphère lagrangienne plongée et un champ de Liouville complet, Comment. Math. Helv. 65 (1990) 623663 MR1078102

[22] K Niederkrüger, The plastikstufe - a generalization of the overtwisted disk to higher dimensions, Algebr. Geom. Topol. 6 (2006) 2473-2508 MR2286033

[23] K Niederkrüger, O van Koert, Every contact manifolds can be given a nonfillable contact structure, Int. Math. Res. Not. (2007) rnm115 MR2380008

[24] F Presas, A class of non-fillable contact structures, Geom. Topol. 11 (2007) 2203-2225 MR2372846

Klaus Niederkrüger, Institut de mathématiques de Toulouse

Université Paul Sabatier - Toulouse III, 31062 Toulouse Cedex 9, France

Francisco Presas, ICMAT, CSIC, Facultad de Matematicas

Universidad Complutense de Madrid, Plaza de Ciencias no 3, 28040 Madrid, Spain

niederkr@math.univ-toulouse.fr, fpresas@imaff.cfmac.csic.es

Proposed: Leonid Polterovich

Seconded: Danny Calegari and Yasha Eliashberg
Received: 19 March 2009

Revised: 11 November 2009 